\title{
hdac4 mediates perichondral ossification and pharyngeal skeleton development in the zebrafish
}

\author{
April DeLaurier ${ }^{\text {Corresp.., }}{ }^{\text {, }}$, Cynthia Lizzet Alvarez ${ }^{2}$, Kali J Wiggins ${ }^{2}$ \\ 1 Department of Biology and Geology, University of South Carolina - Aiken, Aiken, United States \\ 2 Department of Biology and Geology, University of South Carolina - Aiken, Aiken, South Carolina, United States \\ Corresponding Author: April DeLaurier \\ Email address: aprild@usca.edu
}

Background. Histone deacetylases (HDACS) are epigenetic factors that function to repress gene transcription by removing acetyl groups from the $\mathrm{N}$-terminal of histone lysines. Histone deacetylase 4 (HDAC4), a class Ila HDAC, has previously been shown to regulate the process of endochondral ossification in mice via repression of Myocyte enhancer factor 2c (MEF2C), a transcriptional activator of Runx2, which in turn promotes chondrocyte maturation and production of bone by osteoblasts.

Methods \& Materials. In this study, we generated two zebrafish lines with mutations in hdac4 using CRISPR/Cas9 and analyzed mutants for skeletal phenotypes and expression of genes known to be affected by Hdac4 expression.

Results: Lines have insertions causing a frameshift in a proximal exon of hdac4 and a premature stop codon. Mutations are predicted to result in aberrant protein sequence and a truncated protein, eliminating the Mef2c binding domain and Hdac domain. Zygotic mutants show a significant increase in ossification of pharyngeal ceratohyal cartilages at 7 days post fertilization (dpf) $(p<0.01, p<0.001)$. At 4 $\mathrm{dpf}$, mutant larvae have a significant increase of expression of runx $2 a$ and runx $2 b$ in the ceratohyal cartilage ( $p<0.05$ and $p<0.01$, respectively). A subset of maternal-zygotic (mz) mutant and heterozygote larvae (40\%) have dramatically increased ossification at $7 \mathrm{dpf}$ compared to zygotic mutants, including formation of a premature anguloarticular bone and mineralization of the first and second ceratobranchial cartilages and symplectic cartilages, which normally does not occur until fish are approximately 10 or 12 dpf. Some maternal-zygotic mutants and heterozygotes show loss of pharyngeal first arch elements ( $25.9 \%$ and $10.2 \%$, respectively) and neurocranium defects ( $30.8 \%$ and $15.2 \%$, respectively). Analysis of RNA-seq mRNA transcript levels and in situ hybridizations from zygotic stages to 75-90\% epiboly indicates that hdac4 is highly expressed in early embryos, but diminishes by late epiboly, becoming expressed again in larval stages.

Discussion. Loss of function of hdac4 in zebrafish is associated with increased expression of runx2a and run $x 2 b$ targets indicating that a role for hdac4 in zebrafish is to repress activation of ossification of cartilage. These findings are consistent with observations of precocious cartilage ossification in Hdac4 mutant mice, demonstrating that the function of Hdac4 in skeletal development is conserved among vertebrates. Expression of hdac4 mRNA in embryos younger than 256-512 cells indicates that there is a maternal contribution of hdac4 to the early embryo. The increase in ossification and profound loss of first pharyngeal arch elements and anterior neurocranium in a subset of maternal-zygotic mutant and heterozygote larvae suggests that maternal hdac4 functions in cartilage ossification and development of cranial neural crest-derived structures. 
1 hdac4 mediates perichondral ossification and 2 pharyngeal skeleton development in the zebrafish

4 5

6

7

8

9 Corresponding Author:

10 April DeLaurier ${ }^{1}$

11 Dept. of Biology and Geology, University of South Carolina Aiken, 471 University Parkway,

12 Aiken, South Carolina, 29801, USA

13 Email address: aprild@usca.edu 


\section{Abstract}

20 Background. Histone deacetylases (HDACs) are epigenetic factors that function to

21 repress gene transcription by removing acetyl groups from the $\mathrm{N}$-terminal of histone lysines. Histone deacetylase 4 (HDAC4), a class Ila HDAC, has previously been shown to regulate the process of endochondral ossification in mice via repression of Myocyte enhancer factor 2c (MEF2C), a transcriptional activator of Runx2, which in turn promotes chondrocyte maturation and production of bone by osteoblasts.

Methods \& Materials. In this study, we generated two zebrafish lines with mutations in hdac4 using CRISPR/Cas9 and analyzed mutants for skeletal phenotypes and expression of genes known to be affected by Hdac4 expression.

Results: Lines have insertions causing a frameshift in a proximal exon of hdac4 and a premature stop codon. Mutations are predicted to result in aberrant protein sequence

31 and a truncated protein, eliminating the Mef2c binding domain and Hdac domain. Zygotic mutants show a significant increase in ossification of pharyngeal ceratohyal cartilages at 7 days post fertilization (dpf) $(p<0.01, p<0.001)$. At 4 dpf, mutant larvae have a significant increase of expression of runx $2 a$ and $r u n x 2 b$ in the ceratohyal cartilage $(p<0.05$ and $p<0.01$, respectively). A subset of maternal-zygotic (mz) mutant and heterozygote larvae (40\%) have dramatically increased ossification at $7 \mathrm{dpf}$ compared to zygotic mutants, including formation of a premature anguloarticular bone and mineralization of the first and second ceratobranchial cartilages and symplectic cartilages, which normally does not occur until fish are approximately 10 or $12 \mathrm{dpf}$.

40 Some maternal-zygotic mutants and heterozygotes show loss of pharyngeal first arch 41 elements (25.9\% and $10.2 \%$, respectively) and neurocranium defects $(30.8 \%$ and 
$4215.2 \%$, respectively). Analysis of RNA-seq mRNA transcript levels and in

43 situ hybridizations from zygotic stages to $75-90 \%$ epiboly indicates that $h d a c 4$ is highly

44 expressed in early embryos, but diminishes by late epiboly, becoming expressed again 45 in larval stages.

46 Discussion. Loss of function of hdac4 in zebrafish is associated with increased

47 expression of runx2a and runx $2 b$ targets indicating that a role for hdac4 in zebrafish is 48 to repress activation of ossification of cartilage. These findings are consistent with 49 observations of precocious cartilage ossification in Hdac4 mutant mice, demonstrating 50 that the function of $\mathrm{Hdac} 4$ in skeletal development is conserved among vertebrates.

51 Expression of hdac4 mRNA in embryos younger than 256-512 cells indicates that there 52 is a maternal contribution of $h d a c 4$ to the early embryo. The increase in ossification and 53 profound loss of first pharyngeal arch elements and anterior neurocranium in a subset 54 of maternal-zygotic mutant and heterozygote larvae suggests that 55 maternal hdac4 functions in cartilage ossification and development of cranial neural 56 crest-derived structures. 


\section{Introduction}

66 The majority of the vertebrate skeleton including the axial, limb, and pharyngeal

67 elements form as cartilaginous elements that grow rapidly during early development

68 through the proliferation of matrix-secreting chondrocytes (Karsenty \& Wagner, 2002).

At specific stages of development, chondrocytes cease their rapid proliferation and

matrix secretion, become hypertrophic, and signal to nearby cells to commence

endochondral ossification (Karsenty \& Wagner, 2002). In zebrafish, ossification involves

72 the recruitment of osteoblasts to the surface of cartilage to secrete a perichondral collar

73 of mineralized bone (Hall, 2014). In amniotes, endochondral ossification involves both

perichondral ossification and the invasion of the cartilage by blood vessels to deliver

osteoblasts which deposit bone (Hall, 2014). Among vertebrates, the timing of the

transition between endochondral growth and ossification is important in the determining

size and shape of skeletal elements (Nagata et al., 2011; Eames et al., 2011;

Grünbaum, Cloutier \& Vincent, 2012; Harrington, Harrison \& Sheil, 2013; Arenas-

Rodríguez, Rubiano Vargas \& Hoyos, 2018). This process is precisely regulated by

expression of factors in chondrocytes and osteoblasts including Ihh, Pthrp, Runx2

(Cbfa1), and Sp7 (Osx) (Vortkamp et al., 1996; Komori et al., 1997; Nakashima et al.,

2002; Maeda et al., 2007). Loss of function of these factors can cause insufficient

ossification, resulting in severe growth and patterning defects of the skeleton (Quack et

al., 1999; Wysolmerski et al., 2001; Gao et al., 2001; Valadares et al., 2014).

regulator of chondrocyte maturation and initiation of endochondral ossification in mice 
88 (Vega et al., 2004). HDAC4 lacks the ability to bind to DNA directly, but associates with

89 other proteins to remove acetyl groups from the $\mathrm{N}$-terminal of histone lysines, causing

90 histones to condense, blocking access of transcription factors to DNA, resulting in

91 transcriptional repression (Haberland, Montgomery \& Olson, 2009). Class Ila HDACs

92 are characterized by a carboxyl-terminal binding protein domain (CtBP), a Mef2 binding

93 domain for binding the transcription factor MEF2C, sites for binding of the chaperone

94 protein 14-3-3, and an HDAC domain (Haberland, Montgomery \& Olson, 2009). MEF2C

95 is a transcription factor which controls chondrocyte hypertrophy and bone formation by

96 activating target genes such as Runx2 (Arnold et al., 2007). When class lla HDACs are

97 unphosphorylated, they localize to the nucleus where they bind to MEF2C, and function

98 to repress transcription of MEF2C-target genes (Lu et al., 2000; Passier et al., 2000;

99 McKinsey et al., 2000; Arnold et al., 2007). When calcium/calmodulin protein kinase

100 (CaMKII) and protein kinase D (PKD) phosphorylate 14-3-3 and shuttle the Hdac4 into

101 the cytoplasm, MEF2C becomes unbound to Hdac4, and can activate transcription of

102 target genes (Lu et al., 2000; Passier et al., 2000; McKinsey et al., 2000). Through this

103 process of interaction with MEF2C, HDAC4 delays the hypertrophy of chondrocytes

104 within cartilage, controlling the timing and extent of ossification of endochondral bone by

105 osteoblasts (Vega et al., 2004).

106 Zebrafish represent a useful model for studying mechanisms of chondrocyte

107 maturation and the initiation of the cartilage ossification process as they develop

108 cartilaginous elements as early as 60-72 hours post-fertilization and commence

109 perichondral ossification of these elements as early as $96 \mathrm{hpf}$ (Eames et al., 2013).

110 Compared with other vertebrates, zebrafish undergo the same cellular and genetic 
111 signaling pathways associated with skeletal ossification including chondrocyte

112 hypertrophy, differentiation and matrix secretion by osteoblasts, including expression of

113 factors associated with ossification such as ihha, runx2a, runx2b, sp7, col1a2, col10a1,

114 and osteonectin (Flores et al., 2004; Avaron et al., 2006; Li et al., 2009). These

115 similarities with other vertebrates such as mice make zebrafish a useful model to study

116 genetic pathways associated with skeletal development and disease.

117 In this study, we describe two zebrafish lines with early frameshift mutations in

118 hdac4. Mutant larvae from heterozygote intercrosses show an increase in ossification of

119 ventral pharyngeal cartilage elements, and up-regulation of markers of ossification

120 including runx2a and runx2b. A further enhancement of the excessive ossification defect

121 is observed in maternal-zygotic mutants, indicating an early maternal contribution to

122 skeletal patterning in zebrafish. Previously, we identified a potential role for hdac4 in

123 neural crest development and neurocranium formation in zebrafish (DeLaurier et al.,

124 2012). Although this phenotype was not reproduced in any zygotic mutants, a profound

125 loss of first pharyngeal arch facial structures was observed in a subset of maternal-

126 zygotic mutants, indicating a function for maternal hdac4 in neural crest development or

127 formation of other anterior structures of the head or face. In conclusion, hdac4 mutants

128 reproduce aspects of the mouse Hdac4 mutant for cartilage ossification and

129 demonstrate a role for maternal hdac4 in development of the anterior neural crest-

130 derived facial skeleton in zebrafish. This mutant line may be a useful model, especially

131 along with other reverse-genetic mutants for other class lla Hdacs, to study the function

132 of this class of epigenetic regulators in skeletal patterning and other developmental

133 pathways. 


\section{Methods}

136

137 Zebrafish husbandry

138 AB strain wild-type (WT) zebrafish were originally obtained from the Zebrafish

139 International Resource Center (ZIRC, Eugene, OR). Fish were reared and maintained at

$14028.5^{\circ} \mathrm{C}$ on a 14 hour on/10 hour off light cycle. Fish were fed as previously described

141 (Wasden, Roberts \& DeLaurier, 2017). Maintenance and use of zebrafish followed

142 guidelines from ZIRC, the Zebrafish book (Westerfield, 2007), and the Institutional

143 Animal Care and Use Committee (IACUC) of the University of South Carolina Aiken

144 (approval number 010317-BIO-01). Fish were staged using criteria for staging

145 development up to $3 \mathrm{dpf}$ (Kimmel et al., 1995). For $7 \mathrm{dpf}$ samples, at least $95 \%$ of fish

146 within a clutch had to show swim bladders in order to be included in analysis.

\section{Generation of CRISPR lines}

149 The CRISPR/Cas9 procedure was based on previously described methodologies

150 (Hwang et al., 2013). CHOPCHOP (http://chopchop.cbu.uib.no/) was used to design a

151 guide RNA (gRNA) sequence targeting exon 5 of hdac4 (ensembl

152 ENSDART00000165238.3), 5' of the Mef2c binding site (located in exon 6) and histone

153 deacetylase domain, with minimal potential off-target binding (Montague et al., 2014;

154 Labun et al., 2016). An hdac4-specific oligonucleotide was designed containing a 20 bp

155 T7 promoter sequence, 20bp of target sequence (GGAGCGTCATCGACAGGAGC),

156 followed by a 20 bp scaffold overlap sequence as described (Bassett et al., 2013). This 
157 oligonucleotide was annealed to a scaffold oligonucleotide containing the tracrRNA

158 stem loop sequence using Phusion PCR (New England Biolabs, Ipswich, MA) to

159 produce a 120 bp template. Template DNA was column purified (DNA Clean \&

160 Concentrator kit, Zymo Research, Irvine, CA) and was used to synthesize RNA using

161 T7 polymerase (MAXIscript T7 Transcription kit, Thermo Fisher, Vitnus, Lithuania).

162 Cas9 mRNA was synthesized from pCS2-nCas9n (Addgene, Cambridge, MA). The

163 plasmid was linearized with Notl-HF (New England Biolabs, Ipswich, MA), column

164 purified (Zyppy Plasmid Miniprep kit, Zymo Research, Irvine, CA), and mRNA was

165 synthesized (mMessenger mMachine SP6 kit, Thermo Fisher, Vitnus, Lithuania).

166 Column-purified hdac4 gRNA and nCas9n mRNA (RNA Clean \& Concentrator kit, Zymo

167 Research, Irvine, CA) were co-injected into one cell-stage embryos. Each embryo was

168 injected with approximately $3 \mathrm{nl}$ of a $5 \mathrm{ul}$ mix containing hdac4 gRNA ( $60 \mathrm{ng} /$ microliter),

169 nCas9n mRNA ( 160ng/microliter), and phenol red as a marker. Unfertilized or dead

170 embryos were removed from the dish at the end of the first day of injection and on

171 subsequent days.

172

173 Identification of founders and generation of mutant lines

174 At 36 hpf, approximately $20 \%$ of injected embryos (abnormal and normal-looking) were

175 pooled into groups of 5 fish per tube, lysed using HotShot (Truett et al., 2000), and PCR

176 was performed using genomic primers flanking the site of potential mutation. PCR

177 products were gel-purified and digested using T7 endonuclease (New England Biolabs,

178 Ipswich, MA) to identify mismatched DNA indicating potential founder lines (Hwang et

179 al., 2013). Siblings of fish (approximately 40 fish) with positive T7 results were reared to 
180 adulthood and used as founder $\left(F_{0}\right)$ lines for subsequent experiments. Three $F_{0}$ fish

181 demonstrated germ line transmission to offspring, and $F_{1}$ lines were generated from

182 these founders by out-crossing founders to $A B$ wild-types. Adult $F_{1}$ fish were identified

183 as heterozygous carriers of potential mutations using PCR and T7 endonuclease digest

184 on amputated tail DNA. Heterozygous $F_{1}$ fish were outcrossed to generate $F_{2}$ lines, and

$185 \mathrm{~F}_{2}$ lines were intercrossed to produce homozygous mutants. PCR products from

186 potential mutants and wild-type siblings were sequenced (Eurofins, Louisville, KY) and

187 genomic sequences were compared to wild-type siblings to identify mutations

188 (Geneious, version 8, http://www.geneious.com) (Kearse et al., 2012). Mutations were

189 confirmed by synthesis of cDNA from mRNA (RevertAid First Strand cDNA Synthesis

190 kit, Thermo Fisher, Vitnus, Lithuania) and then amplified by PCR using an exon 3

191 forward primer 5'-gccactggaacttctcaagc-3' and an exon 6 reverse primer 5'-

192 gcagtggttgagactcctct- $3^{\prime}\left(\mathrm{Tm}=58^{\circ} \mathrm{C} \times 40\right.$ cycles or Touchdown PCR, $\mathrm{Tm}=72-65^{\circ} \mathrm{C} \times 15$

193 cycles followed by $\mathrm{Tm}=64.5^{\circ} \mathrm{C} \times 20$ cycles). $\mathrm{PCR}$ products were column purified as

194 described above and sequenced to confirm the mutation. Heterozygous $F_{2}$ and $F_{3}$

195 carriers of mutant alleles were intercrossed to produce wild-type, heterozygote, and

196 mutant offspring. In order to test for the influence of maternal hdac4 on development,

197 maternal-zygotic mutants were generated using the $h d a c 4^{\text {aik3 }}$ line by crossing

198 homozygote mutant females with heterozygote males. Female mutants could not be

199 generated using the hdac4aik2 line, although mutant males survived.

200

201 Genotyping adults and larvae 
202 Amputated tails from adult fish, amputated tails from stained larvae, or whole larvae

203 were genotyped using Hotshot lysis as described above. An 822bp region of exon 5

204 spanning the site of mutation in hdac4 was PCR-amplified using an intron 4-5 forward

205 primer 5'-atgttctccctgtgttggtg-3' and an intron 5-6 reverse primer 5'-gctgtatttccgctcatgtg-

$2063^{\prime}\left(\mathrm{Tm}=58^{\circ} \mathrm{C}, \mathrm{x} 40\right.$ cycles $) . \mathrm{PCR}$ products were run on a $2 \%$ agarose gel at $60 \mathrm{~V}$ for $5-6$

207 hours to produce band separation sufficient to distinguish heterozygote (2 bands) fish

208 from wild-type and mutant fish (both 1 lower and upper band, respectively).

210 Alcian Blue and Alizarin Red histological stain

211 Alcian Blue and Alizarin Red staining to label cartilage and bone was performed as

212 described (Walker \& Kimmel, 2007). Briefly, 7 dpf zebrafish were fixed for 1 hour in $2 \%$

213 paraformaldehyde/1X PBS, washed in $50 \%$ ethanol $/ \mathrm{H}_{2} \mathrm{O}$, and stained overnight rocking

214 at room temperature in $0.04 \%$ Alcian Blue (Anatech, Battle Creek, MI)/0.01\% Alizarin

215 Red S (Sigma-Aldrich, St. Louis, MO)/10mm MgCl$/ 20 \%$ ethanol. On day 2, fish were

216 rinsed in $80 \%$ ethanol $/ 10 \mathrm{mM} \mathrm{MgCl}_{2} / \mathrm{H}_{2} \mathrm{O}$ for several hours, then washed in $50 \%$ and

$21725 \%$ ethanol $/ \mathrm{H}_{2} \mathrm{O}$, bleached using $3 \% \mathrm{H}_{2} \mathrm{O}_{2} / 0.5 \% \mathrm{KOH}$ for 10 minutes, then cleared in

$21825 \%$ glycerol $/ 0.1 \% \mathrm{KOH}$, and incubated in $50 \%$ glycerol $/ 0.1 \% \mathrm{KOH}$ overnight, rocking at

219 room temperature. Skeletal preparations were stored covered at 4 degrees Celcius.

220

221 mRNA in situ hybridization

222 Single non-fluorescent and double fluorescent mRNA in situ hybridizations were

223 performed as described (Talbot, Johnson \& Kimmel, 2010; Thisse \& Thisse, 2014),

224 using probes for hdac4, runx2a, runx2b, sp7, and sox9a (DeLaurier et al., 2010; 
225 Huycke, Eames \& Kimmel, 2012) using larvae from the hdac4aik3 line. Fish were

226 genotyped after staining. All mutant and wild-type larvae were used for confocal imaging

227 and image analysis. No larvae were excluded from analysis with the exception of larvae

228 that were damaged during processing or imaging. For mRNA in situ hybridizations of

229 embryos from 2-cell to $90 \%$ epiboly stages, all embryos were collected from the same

230 clutch of fertilized eggs on the same day, and fixed in $4 \%$ paraformaldehyde at specific

231 stages. All embryos were pooled for in situ hybridization and developed for staining

232 simultaneously. The reaction was stopped at the same point for all specimens.

233 Specimens used for in situ hybridization were stored covered, in 1X PBS, at 4 degrees

234 Celcius.

235

236 Imaging, image analysis, and statistics: skeletal preparations

237 Alcian Blue and Alizarin Red stained specimens were dissected and flat mounted on

238 microscope slides and imaged on a compound microscope. The right pharyngeal

239 skeleton was flat mounted for each specimen unless it was damaged or defective, in

240 which case the left side was used. Among all genotyped fish, the extent of ossification

241 of the ceratohyal was scored after removing sample identification and genotype

242 information to ensure unbiased analysis. The region of ossification was scored for the

243 evenness of the border of Alizarin red stain (regular border, irregular border), and for the

244 approximate proportion of total area of the element that was stained (small area $=$ less

245 than approximately $20 \%$ or less of the total area, large area $=$ greater than

246 approximately $20 \%$ of the total area). In total, 94 fish were scored for the $h d a c 4^{\text {aik2 }}$ line 
247 (39 wild-type, 23 heterozygotes, and 32 mutants), and 96 fish were scored for the 248 hdac4 $^{\text {aik3 }}$ line (22 wild-type, 44 heterozygotes, and 30 mutants).

249 In order to quantitate the levels of ossification in wild-type, heterozygous, and

250 mutant fish, the areas of flat mounted ceratohyal and hyosymplectic cartilages were

251 measured using pixel area of cartilage and bone using Image $1.51 \mathrm{~m} 9$ (Schneider,

252 Rasband \& Eliceiri, 2012). In total, 93 fish were measured for the hdac4 $4^{\text {aik2 }}$ line (38 wild-

253 type, 23 heterozygote, 32 mutant) and 61 fish were measured for the hdac4 $4^{\text {aik3 line (17 }}$

254 wild-type, 28 heterozygote, 16 mutant). The area of bone as a ratio of cartilage area

255 was used a measure of ossification in statistical analysis (SAS 9.4, SAS Institute,

256 Carey, NC). Total area of the element was used as a method to control for differences

257 in ossification due to subtle growth/stage differences between individual fish. For the

$258 h^{2}$ ac4 $^{\text {aik2 }}$ line, for the ceratohyal, and for the hdac4aik3 line, for the hyosymplectic,

259 measurements of "bone area" and "total area" met assumptions of normality and

260 homogeneity of slope assumptions for analysis of covariance (ANCOVA). For the

261 hdac4 $4^{a i k 2}$ line, for the hyosymplectic, and for the hdac4aik3 line for the ceratohyal, there

262 was no significant relationship between measurements of "bone area" and "total area"

263 and so there was no reason to apply ANCOVA, and conventional ANOVA was applied.

264 In the case of application of ANOVA, datasets met assumptions of normality and equal

265 variance between comparison groups (Shapiro-Wilk normality test, Hartley's $F_{\text {max }}$-test,

266 SAS 9.4, SAS Institute, Carey, NC).

267

268 Imaging, image analysis, and statistics: mRNA in situ hybridizations 
269 Specimens used for fluorescent in situ hybridization were mounted ventral-side down on

270 glass coverslips and imaged using an inverted Leica SPEII confocal microscope (Leica

271 Microsystems, Buffalo Grove, IL). In total, 21 4dpf larvae stained using the runx2a probe

272 were imaged (10 wild-type, 11 mutant), $174 \mathrm{dpf}$ larvae stained using the runx $2 b$ probe

273 were imaged ( 8 wild-type, 9 mutant), and $224 \mathrm{dpf}$ larvae stained using the $s p 7$ probe

274 were imaged (12 wild-type, 10 mutant). For runx2a, larvae from two stage-matched

275 clutches were combined for analysis. For runx $2 b$ and $s p 7$, only siblings from a single

276 clutch were analyzed. Each sample was scanned to produce an approximately $80 \mathrm{z}$ -

277 slice stack. Confocal settings were kept exactly the same between imaging sessions.

278 After scanning of all samples was complete, stacks were renamed (removing sample

279 identification and genotype information) and randomized by a participant not involved in

280 this study to ensure unbiased analysis of data. For all image stacks, gene expression

281 was measured as the maximum length of expression (in microns) on the anterior and

282 posterior margins of the right and left ceratohyal cartilages (Leica Application Suite X

283 (LAS X) software 1.8.0.13370, Leica Microsystems). Measurements were recorded

284 twice, on separate days, and data from the two sessions were averaged to produce a

285 final measurement for each region (i.e. left posterior, left anterior, right posterior, right

286 anterior) of the ceratohyal for each sample. All four regions were averaged to produce a

287 final average length of expression of each gene for each sample. Measurements for

288 each genotype within each gene study were assessed for normality and equal variance

289 (Shapiro-Wilk normality test, Hartley's F $_{\max }$-test, SAS 9.4, SAS Institute, Carey, NC). All

290 datasets met assumptions of normality and groups that were compared had equal

291 variance, so ANOVA was performed. 


\section{RNA-Seq data}

294 We used the RNA-seq expression atlas data for zebrafish

295 (https://www.ebi.ac.uk/gxa/experiments/E-ERAD-475/Results) to establish expression of 296 hdac4 transcripts per million transcripts (TPM) between zygote (1-cell) and 5dpf-stage 297 larvae.

298

\section{Results}

300 Hdac4 mutants have a frameshift lesion

301 Using CRISPR/Cas9 to target exon 5 of hdac4 (Fig. 1A), we induced frameshift

302 mutations in two individual fish that were used to generate mutant lines. The hdac4aik2

303 allele has a $19 \mathrm{bp}$ insertion three bases upstream of the protospacer adjacent motif

304 (PAM) site associated with Cas9 binding and cleaving of DNA. The hdac4aik3 allele has

305 a 2 bp insertion, followed by retention of $7 \mathrm{bp}$ of the wild-type sequence, followed by a

30627 bp insertion one base pair upstream of the PAM site (Fig. 1B). In both cases,

307 frameshifts were induced by insertion of nucleotides into exon 5, resulting in aberrant

308 amino acids being added to the protein sequence (Fig. 1C). In both mutant lines the

309 frameshift is predicted to cause the loss of the Mef2c binding domain and premature

310 stop codons resulting in truncated proteins 174 aa (hdac4aik2) and 181 aa (hdac4aik3) in

311 length (Fig. 1D). Frameshifts were detected in mutant cDNA compared to wild-type

312 cDNA using primers spanning exons 3-6, and there was no evidence of splice variants

313 or exon skipping detected on agarose gels for either mutant (Figs. 1E and F). In the

314 case of hdac4 ${ }^{\text {aik3 }}$, a larger band was detected along with the band of expected size (Fig. 
$3151 \mathrm{~F}$, indicated by asterisk). This band was excised and sequenced and was found to

316 have an identical sequence to the band of the expected size. We interpret that this

317 larger band is the product of heterodimers of our PCR product or a slower running

318 single-stranded DNA product and not a splice variant or other genomic feature within

319 the mutant. In both the hdac4 ${ }^{a i k 2}$ and $h d a c 4^{a i k 3}$ lines, adult fish and embryos were

320 genotyped using intronic primers spanning exon 5 . The larger mutant band (841 bp

321 hdac4 $4^{a i k 2}, 858 \mathrm{bp}$ hdac4 ${ }^{a i k 3}$ ) can be distinguished from the wild-type band (822bp), and

322 heterozygotes show both bands (Figs. $1 \mathrm{G}$ and $\mathrm{H}$ ). At $7 \mathrm{dpf}$ and earlier, hdac4aik3 mutants

323 have no discernable external abnormalities compared to wild-type siblings (Figs. $1 \mathrm{I}$ and

324 J). Mutant fish form swim bladders, feed, and grow normally into adult fish. Mutant fish

325 from the hdac4 ${ }^{a i k 2}$ line also showed no overt external abnormalities compared to wild-

326 type siblings (data not shown).

327

\section{Mutants have increased ossification of pharyngeal cartilage}

329 Examination of Alcian Blue and Alizarin Red-stained specimens reveal that mutants

330 from the hdac4 ${ }^{a i k 2}$ and $h d a c 4^{a i k 3}$ lines showed a greater extent of ossification of the

331 ceratohyal cartilage compared to wild-type siblings (Fig. 2B-E', hdac4aik2 only shown in

332 D-E', hdac4 ${ }^{a i k 3}$ not shown). Wild-type fish had a smaller area of bone stain localized to

333 the mid-shaft of the ceratohyal, usually appearing first at the dorsal margin of the

334 cartilage and spreading ventrally, and this region of bone had even, regular, borders

335 (Fig. 2D and D', see Figs. 2B and C for frequencies). Among mutants, a larger area of

336 bone was observed at the mid-shaft of the ceratohyal, and this area of bone showed

337 irregular borders (Fig. 2E and E', see Figs. 2B and C for frequencies). Among 
338 heterozygotes, the amount of bone area on the ceratohyal appeared as a mixture of the

339 small and large areas observed in wild-types and mutants, and showed both regular

340 and irregular borders (not shown, see Figs. 2B and C for frequencies). No other defects

341 were detected in the pharyngeal skeleton or neurocrania in larvae analyzed at this

342 stage.

343 For both the ceratohyal and hyosymplectic, the area of bone and the area of

344 cartilage were measured, and the amount of ossification was calculated as the ratio of

345 bone to cartilage present (Figs. 2F-I). For the hdac4aik2 line, ANCOVA revealed that the

346 effect of genotype on the area of ossification of the ceratohyal was significant $(F=4.01$;

$347 \mathrm{df}=2,89 ; \mathrm{p}=0.0215)$. Tukey's multiple comparisons showed that mutants had

348 significantly more bone than wild-types $(p=0.0057)$, but heterozygotes were not

349 significantly different from either mutants $(p=0.1751)$ or wild-types $(p=0.2488)$ (Fig. $2 F)$.

350 For the hdac4 ${ }^{a i k 3}$ line, ANOVA revealed that the effect of genotype on the area of

351 ossification of the ceratohyal was also significant $(F=7.77 ; d f=2,58 ; p=0.001)$. Tukey's

352 multiple comparisons showed that mutants had significantly more bone than wild-types

$353(p=0.0002)$, that heterozygotes also had significantly more bone than wild-types

$354(p=0.0134)$, and that there was no significant difference between heterozygotes and

355 mutants ( $p=0.0697)$ (Fig. 2G). For the hdac4 $4^{a i k 2}$ line, ANOVA revealed no significant

356 effect of genotype on area of ossification of the hyosymplectic $(F=2.3 ; d f=2,90 ; p=0.106)$

357 (Fig. 2H). For the hdac4aik3 line, ANCOVA also revealed no significant effect of

358 genotype on area of ossification of the hyosymplectic $(F=2.48 ; d f=1,56 ; p=0.0928)$ (Fig. 359 2I). 
361 hdac4 is expressed in regions of the pharyngeal skeleton consistent with a role in

362 cartilage maturation

363 mRNA in situ hybridization was used to detect hdac4 transcripts in larvae at 72 hpf.

364 Previously, we described the expression of hdac4 in the ventral region of the developing

365 pharyngeal skeleton at 72 hpf (DeLaurier et al., 2012), and here we show how specific

366 regions of expression are associated with sites of ossification of cartilage. By $72 \mathrm{hpf}$,

367 expression is localized to regions of sox9a-expressing cartilage as well as in tissue

368 surrounding cartilage and dermal bone elements. Co-expression of hdac4 and sox9a

369 was detected in the hyosymplectic, ceratohyal, and palatoquadrate cartilages (Figs. 3A-

$370 \mathrm{H}$, indicated by arrows). In the case of the hyosymplectic and ceratohyal, co-expression

371 of hdac4 and sox9a was in regions that undergo ossification at later stages. hdac4 was

372 strongly expressed in the posterior pharyngeal arches, overlapping in the mid-region of

373 each arch with a domain of sox9a expression in the ceratobranchial cartilage within

374 each arch (Figs. 3I-L).

375

376 Mutants have increased expression of runx2 factors

377 MEF2C is known to activate transcription of Runx2, a transcription factor that activates

378 chondrocyte maturation (Arnold et al., 2007). Runx2 in turn activates Sp7, a

379 transcription factor associated with osteoblast differentiation (Nishio et al., 2006). We

380 examined how loss of hdac4, which would lead to over-activity of Mef2c, affects levels

381 of mRNA expression of these factors. Among teleost fish, a whole genome duplication

382 event generated two copies of vertebrate runx2, runx2a and runx2b (van der Meulen et

383 al., 2005). As in previous studies, we observed differential patterns of expression of 
384 both genes, indicating that these genes have distinct functions in skeletal development

385 in zebrafish (Figs. 4A-H). At $4 \mathrm{dpf}$, runx2a and runx2b were expressed in the mid-shaft

386 region of the ceratohyal cartilage and branchiostegal ray (Fig. 4B and F), and also in the

387 opercle (not shown). In general, runx2a expression was broader than runx $2 b$ expression

388 at $4 \mathrm{dpf}$, encompassing more of the anterior and posterior lengths of the ceratohyal

389 (Figs. 4B and F). runx2b was expressed in the hyosymplectic at $4 \mathrm{dpf}$, but runx2a was

390 not (not shown). Among mutants, the average length of runx2a expression in the

391 ceratohyal was significantly increased compared to wild-types (Fig. 4A-D, indicated by

392 arrows, $\mathrm{O} ; \mathrm{F}=6.49 ; \mathrm{df}=1,19 ; \mathrm{p}=0.0196)$. Expression of $r u n \times 2 b$ was also significantly

393 increased in mutants compared to wild-types (Fig. 4E-H, indicated by arrows, P;

$394 \mathrm{~F}=11.94 ; \mathrm{df}=1,15 ; \mathrm{p}=0.0035)$. At $4 \mathrm{dpf}$, in both wild-types and mutants, sp7 was

395 expressed in a small patch of cells on the posterior margin, and less frequently also on

396 the anterior margin, of the mid-shaft of the ceratohyal in both wild-type and mutant

397 larvae (Figs. 4I-L, indicated by arrows). Analysis of the average length of $s p 7$

398 expression revealed no significant differences between mutants and wild-types (Fig. 4Q,

$399 \mathrm{~F}=0.0001 ; \mathrm{df}=1,20 ; \mathrm{p}=0.99)$. Schematic diagrams showing the differences in patterns of

400 expression of runx2a, runx $2 b$, and $s p 7$ in wild-type vs. zygotic mutant larvae at $4 \mathrm{dpf}$ are

401 shown in Figures $4 \mathrm{M}$ and $\mathrm{N}$.

402

403 Maternal-zygotic mutants have increased ossification of the pharyngeal skeleton

404 and defects in the anterior facial region

405 In order to examine the role of a maternal contribution of hdac4 to development, we

406 performed analyses of skeletal phenotypes in mutants and heterozygotes generated 
407 from maternal mutants crossed with heterozygote males. At $7 \mathrm{dpf}$, a subset of maternal-

408 zygotic mutants and heterozygotes showed a prominent increase in cartilage

409 ossification compared to wild-types (non-sibling controls) (Figs. 5A, C-E, G,I). In total,

$41040.0 \%$ (16/40) of maternal-zygotic mutants and 40.0\% (16/40) heterozygotes had

411 evidence of premature or excessive ossification (Fig. 5F), and resembled ossification

412 patterns present in $12 \mathrm{dpf}$ wild-type fish (Fig. 5B). Increased ossification in mutants and

413 heterozygotes included formation of an anguloarticular bone associated with the

414 Meckel's cartilage (Figs. 5B-D), an enlarged quadrate (Figs. 5C and E), a ventral

415 hypohyal element associated with the ceratohyal (Figs. 5C and E), and ossification of

416 the symplectic cartilage (Fig. 5C, indicated by asterisk). Among these maternal-zygotic

417 mutants and heterozygotes, ossification of the mid-shaft of the first or first and second

418 ceratobranchial cartilages was detected, which is also normally observed in wild-type

419 larvae at $12 \mathrm{dpf}$ (Figs. 5D, E, G-I).

420

421 Maternal-zygotic mutants have defects in the development of first pharyngeal

422 arch skeletal elements and the anterior neurocranium

423 Among maternal-zygotic mutants, $25.9 \%(15 / 58)$ had defects of the first pharyngeal

424 arch skeleton, including a shortened face (Figs. 6A and B, G) and loss of one or both

425 Meckel's cartilages and the anterior portion of the palatoquadrate cartilage, with

426 retention of a small remnant of the entopterygoid and quadrate (Fig. 6C, see Figs. 2A

427 and $\mathrm{D}$ for reference). Among maternal-zygotic heterozygotes, 10.2\% (6/59, Fig. 6G)

428 also had loss of first pharyngeal arch structures. In the case of larvae with a loss of first

429 arch cartilages, posterior second arch and more posterior arch structures including the 
430 ceratohyal, branchiostegal ray, and opercle were present (Fig. 6C). Some maternal-

431 zygotic mutants and heterozygotes had defects in the neurocranium cartilage (the

432 primary palate in fish), including clefts, holes, and shortening of the anterior portion of

433 the element (Fig. 6D representative heterozygote with normal neurocranium, E and F

434 heterozygote and mutant with neurocranium defects). In total, $30.8 \%(16 / 52)$ of

435 maternal-zygotic mutants, and $15.2 \%(7 / 46)$ of maternal-zygotic heterozygotes had

436 neurocranium defects (Fig. 6G). Most neurocranium defects occurred in fish that also

437 had loss of first arch structures $(11 / 16,68.8 \%$ of maternal-zygotic mutants, and $5 / 7$,

$43871.4 \%$ of maternal-zygotic heterozygotes).

440 hdac4 transcripts are detected in embryos younger than $\mathbf{5 0} \%$ epiboly

441 Based on the RNA-seq expression atlas data for zebrafish, the highest level of hdac4

442 mRNA expression (relative to total transcripts) is between cleavage (2 cell) and blastula

443 dome (over $1 \mathrm{~K}$ cell stage), followed by relatively low expression of transcripts up to 3

444 dpf (larval protruding mouth), after which transcript levels increase (Fig. 7A). mRNA in

445 situ hybridization experiments detected high levels of hdac4 transcripts in wild-type

446 embryos 512 cells or younger (Figs. 7B-D, stages in between those presented also

447 showed comparable expression). By $75-90 \%$ epiboly, hdac4 expression was either not

448 detectable, or expressed at very low levels compared to younger stages.

\section{Discussion}

451 In this study, we generated two novel zebrafish lines with germ line mutations near the

452 start of hdac4. Both lines have insertions that cause frameshifts and premature stop 
453 codons, which we predict causes truncated proteins with no functional Mef2c binding

454 domain or Hdac domain. Although a CtBP domain may exist in a truncated form of the

455 mutant protein, we do not think this is sufficient to preserve function of the Hdac4

456 protein as a repressor of ossification in the absence of the Mef2c and Hdac domains.

457 PCR and sequencing of cDNA does not reveal any alternative splice forms of the

458 transcript, so we do not believe there is evidence for alternate versions of this protein

459 caused by exon skipping or alternative splicing (Sharpe \& Cooper, 2017).

$460 \quad$ Analysis of zygotic mutants revealed a statistically significant increase in

461 ossification of the ceratohyal cartilage in both lines examined. One line showed a trend

462 towards increased ossification of the hyosymplectic (hdac4aik2), although the increase in

463 bone was not significant in mutants compared to wild-type siblings. Normally, the

464 hyosymplectic is the first element to commence ossification at 4-5 dpf, followed by the

465 ceratohyal by $6 \mathrm{dpf}$ (Eames et al., 2013). In the case of the ceratohyal, ossification

466 normally begins on the anterior margin of the mid-shaft of the element and extends

467 posteriorly and along the length of the cartilage element until around $12 \mathrm{dpf}$ when the

468 proximal end of the ceratohyal ossifies to form the epihyal (Cubbage \& Mabee, 1996;

469 Eames et al., 2013). Previous studies in the Hdac4 mutant mouse show precocious

470 ossification of the endochondral skeleton, with particular enhancement of ossification of

471 chondral rib elements and limb cartilages in newborns (Vega et al., 2004). In both the

472 mouse and the zebrafish, ossification of elements is not ectopic, but rather reveals a

473 premature onset of the ossification process. We detected that ossification was

474 increased in heterozygote offspring (Fig. 2) compared to wild-types (hdac4aik2

475 heterozygotes not significantly different to wild-types, hdac4 ${ }^{a i k 3}$ heterozygotes 
476 significantly different to wild-types). This phenotype in heterozygotes suggests that

477 copies of both alleles of $h d a c 4$ are required in zebrafish to repress ossification in the 478 skeleton.

479 In mouse Hdac4 mutants, Runx2 expression is increased in cartilage, and is 480 associated with the increase of endochondral ossification of elements (Vega et al., 481 2004). In zebrafish, orthologs of runx2, runx2a and runx2b are both expressed in 482 embryonic and larval cartilage and bone, consistent with a function in ossification 483 (Flores et al., 2004; Li et al., 2009; van der Velden et al., 2013). At $48-96 \mathrm{hpf}$, runx2a is 484 expressed in the maxilla, dentary, ceratohyal, opercle, and branchiostegal ray elements 485 of the pharyngeal skeleton (Flores et al., 2004; Li et al., 2009; van der Velden et al., 486 2013). At $48-96 \mathrm{hpf}$, runx $2 b$ is expressed in the ceratohyal, hyosymplectic, 487 parasphenoid, entopterygoid, opercle, branchiostegal ray, and ceratobranchial cartilages of the third to seventh pharyngeal arches (Flores et al., 2004; Li et al., 2009; van der Velden et al., 2013). We observed significantly increased expression of runx2a and runx $2 b$ in the ceratohyal of $h d a c 4$-mutant zebrafish compared to wild-types, which we speculate is due to a de-repression of Mef2c function leading to increased transcription of runx2 paralogs. Phylogenetic analysis indicates that runx2a and runx $2 b$ are divergent paralogs, and thus may be regulated by different factors in zebrafish (van 494 der Meulen et al., 2005). In the case of our study, both runx2a and runx2b appear to be targets of repression by $h d a c 4$, and are not differentially affected by loss of $h d a c 4$. We 496 did not detect any significant changes in $s p 7$, a downstream target of runx2, in the ceratohyal of hdac4 mutants compared to wild-types. Normally, sp7 mRNA expression

498 in the ceratohyal begins around 4dpf (Li et al., 2009; Hammond \& Schulte-Merker, 
499 2009; DeLaurier et al., 2010). A possible reason why we did not detect significant

500 differences between wild-types and mutants at this stage is because expression was

501 still at early stages of initiation. Ideally, we would like to analyze $s p 7$ expression in older

502 embryos ( $5 \mathrm{dpf}$ or older). Unfortunately, in the present study we could not achieve

503 adequate penetration of probe in whole mount samples at stages older than $4 \mathrm{dpf}$ to

504 assess $s p 7$ expression in the ceratohyal. Future studies will investigate $s p 7$ expression

505 in older mutants and in maternal-zygotic mutants using a transgenic reporter for $s p 7$

506 (DeLaurier et al., 2010) or using mRNA in situ hybridization using cryosections.

507 Maternal-zygotic mutants and heterozygotes showed an enhancement of the

508 ossification phenotype observed in zygotic mutants. At $7 \mathrm{dpf}$, these larvae had evidence

509 of a ventral hypohyal at the distal portion of the ceratohyal, ossification of the symplectic

510 cartilage of the hyosymplectic element, and ossification of the anterior first and second

511 ceratobranchial cartilages cartilages. Ossification of the dorsal hypohyal occurs by 10

$512 \mathrm{dpf}$, and ossification of the symplectic and first and second ceratobranchial cartilages

513 occurs around 12-13 dpf (Cubbage \& Mabee, 1996; Eames et al., 2013). The presence

514 of ossification of these elements as early as $7 \mathrm{dpf}$ indicates that the ossification program

515 is accelerated in maternal-zygotic mutants and heterozygotes. Among maternal-zygotic

516 mutants and heterozygotes, the presence of an anguloarticular bone associated with

517 the Meckel's cartilage was also observed. In our hands using Alcian Blue and Alizarin

518 Red staining, and in other studies, this element is normally detected in wild-type fish by

$51912 \mathrm{dpf}$ (Cubbage \& Mabee, 1996). Other, more sensitive confocal imaging of Alizarin

520 Red fluorescence has detected this element as early as $8 \mathrm{dpf}$ (Eames et al., 2013).

521 Unlike the other elements showing premature ossification in maternal-zygotic mutants, 
522 this element is a dermal bone that forms without a cartilaginous precursor (Cubbage \&

523 Mabee, 1996). Other dermal bones appear to be unaffected in maternal-zygotic hdac4

524 mutants, and dermal bones are reported to be unaffected in Hdac4 mutant mice (Vega

525 et al., 2004). We cannot explain why this particular dermal bone appears prematurely in

526 hdac4 mutants; however, as it forms on the surface of the Meckel's cartilage,

527 anguloarticular precursor cells may be responding to signals from the underlying

528 Meckel's cartilage to commence formation of bone. During preparation of this

529 manuscript it was noted that the cartilage of maternal-zygotic mutants and

530 heterozygotes with excessive ossification generally had weaker Alcian Blue stain, and

531 chondrocytes appeared rounder and less well organized compared to wild-type controls

532 (Figs. 5C and D). These differences in chondrocyte morphology and cartilage matrix

533 indicates that Hdac4 may repress chondrocyte hypertrophy in zebrafish.

534 The profound increase in ossification in a subset of maternal-zygotic mutants and 535 heterozygotes compared to zygotic mutants suggests that there is a maternal influence

536 on the chondral ossification program in zebrafish through expression of hdac4. In

537 zebrafish, the maternal-zygotic transition (MZT) commences around 2 hpf (128 cells)

538 where maternal transcripts are degraded and the first waves of zygotic transcripts are

539 generated (Tadros \& Lipshitz, 2009). Since the high levels of hdac4 mRNA in embryos

540 prior to 128 cells stage can only be maternal transcripts, we believe this indicates that

541 there is a role for maternal hdac4 in development. From our experiments, it is unclear

542 how early maternal hdac4 may be affecting ossification of cartilage several days post-

543 fertilization. However, it is possible that maternal Hdac4 protein is still present in cells

544 several days following the MZT and can influence ossification, or alternately, maternal 
545 hdac4 may establish an epigenetic environment or signaling cascade in early embryos

546 which has consequences on downstream skeletogenesis. A recent study has detected

547 maternally-derived proteins in larvae up to at least $10 \mathrm{dpf}$ (Boer et al., 2015), so it is

548 possible that maternally-derived hdac4 may regulate ossification processes in zebrafish

549 up to $7 \mathrm{dpf}$. Future experiments will establish the levels of maternal hdac4 transcripts or

550 protein in zebrafish embryos, and we will examine the function of maternal transcripts or

551 proteins on skeletal development in larvae.

552 Previously, we reported that morpholino knockdown of hdac4 causes loss of

553 neural crest and neurocranium defects in zebrafish (DeLaurier et al., 2012). Our zygotic

554 mutants do not show any evidence of this phenotype. However, approximately one

555 quarter of hdac4 maternal-zygotic mutants show anterior neurocranium defects and a

556 loss of first pharyngeal arch cartilages consistent with a role for hdac4 in neural crest

557 development. This phenotype is strikingly similar to a phenotype described for the

558 zebrafish maternal-zygotic mutants for fascin1a (fscn1a), which have abnormalities in

559 filopodia of a subset of cranial neural crest cells, causing migration defects resulting in a

560 loss of the Meckel's cartilage and anterior palatoquadrate cartilage (Boer et al., 2015).

561 Intriguingly, only approximately $20 \%$ of maternal-zygotic fscn1a mutants show this

562 phenotype, similar to the $25.9 \%$ penetrance observed in hdac4 maternal-zygotic

563 mutants. In the case of $h d a c 4$, the proportion of approximately one quarter of maternal-

564 zygotic mutants with defects suggests there may be effects of other unknown loci,

565 modifiers, or interacting genes that are influencing the phenotypic outcome of loss or

566 reductions of hdac4 on neural crest development. 
The observation in the previous study (DeLaurier et al., 2012) that knockdown of

568 hdac4 affects neural crest patterning is complicated to explain in the context of the

569 neural crest defect detected in maternal-zygotic hdac4 mutants. In the case of the

570 fscn1a mutant study, a phenotype similar to fscn1a maternal-zygotic mutants was

571 observed using a morpholino knockdown approach (Boer, Jette \& Stewart, 2016). In this

572 study, detailed analysis revealed that the fscn1a-morphant phenotype was not identical

573 to the mutant, leading the authors to conclude that morpholinos can induce non-specific

574 artifacts in neural crest cell migration and survival, which can be misinterpreted as a

575 specific neural crest phenotype. Although Boer et al., 2016 used a translation-blocking

576 morpholino (thus targeting maternal and zygotic transcripts) and DeLaurier et al., 2012

577 used a splice-blocking morpholino (targeting only zygotic transcripts) we speculate that

578 the defects observed in hdac4-morphants were due to sensitivity of cranial neural crest

579 cells to morpholinos causing non-specific defects cell migration and survival, producing

580 a phenotype of loss of the anterior neural crest-derived pharyngeal skeleton.

581 Intriguingly, a recent study using a translation-blocking morpholino targeting hdac4

582 reports partial clefting of the orofacial region of injected zebrafish (Rothschild et al.,

583 2018). We believe the phenotype reported in DeLaurier et al. 2012 is the result of

584 morpholino-induced artifacts producing non-specific defects to cell migration and

585 survival. We believe this is the only explanation for the presence of a neural crest

586 phenotype in hdac4-morphants (reported in DeLaurier et al., 2012) and a lack of a

587 neural crest phenotype in zygotic mutants. Furthermore, because the morpholino could

588 not target maternal transcripts, we believe the maternal contribution was unaffected in

589 morpholino knockdowns, further reinforcing the idea that the hdac4 morphant 
590 phenotype reported by DeLaurier et al. 2012 may be the product of a non-specific

591 artifact. Despite the complicating evidence of morpholino data in the light of the current

592 study, we believe that the mutant line presented here reveals a novel function for

593 maternal hdac4 in neural crest development.

$594 \quad$ Based on our findings in maternal-zygotic hdac4 mutants and heterozygotes, our

595 future experiments aim to characterize the cellular defect in maternal-zygotic mutants.

596 We will establish if the loss of anterior facial structures is due to a failure of cranial

597 neural crest cell migration or another defect such as specification of neural crest cells,

598 or defects in the patterning of neural crest-derived skeletal elements. We will also

599 establish the levels of specifically maternal hdac4 in zebrafish embryos and we will

600 examine the function of maternal transcripts or proteins on neural crest migration and

601 patterning of skeletal elements in embryos and larvae.

602

603 Conclusions

604 In conclusion, this study shows that mutation of hdac4 in zebrafish causes premature 605 ossification of the pharyngeal skeleton, consistent with previous findings in the mouse

606 (Vega et al., 2004), indicating a conserved function for Hdac4 among vertebrates.

607 Mutants have increased expression of the transcription factors runx2a and runx $2 b$, 608 activators of the skeletal ossification program, which we speculate are upregulated in

609 response to increased activity of Mef2c through loss of hdac4. Maternal-zygotic

610 crosses, along with RNA-seq analysis and detection of high levels of hdac4 mRNA in

611 512-cell and younger embryos indicate that maternal $h d a c 4$ is an important contributor

612 to embryonic and larval development. A subset of maternal-zygotic mutants and 
613 heterozygotes show an enhancement of the premature ossification defect observed in

614 zygotic mutants. A subset of maternal-zygotic mutants and heterozygotes show

615 profound loss of the anterior facial skeleton, including loss of the anterior neurocranium

616 and first pharyngeal arch-derived elements. Although this study did not determine the

617 underlying mechanism for loss of neural crest-derived skeletal elements in maternal-

618 zygotic mutants and heterozygotes, the striking similarity of the hdac4 mutant with the

619 fscn1a mutant (Boer et al., 2015) suggests loss of hdac4 may be associated with a

620 migration defect in a subset of cranial neural crest cells.

621

\section{Acknowledgements}

623 We would like to thank Jared Talbot, Nathan Hancock, and Alec Jones for providing

624 feedback on drafts of this manuscript, Derek Zelmer and Virginia Shervette for

625 assistance with the statistical analysis, and the Busch-Nentwich lab for providing RNA-

626 seq data.

627 
628 Figure legends

629

630 Figure 1: Overview of CRISPR strategy and generation of hdac4 mutant lines. A:

631 Genomic structure of hdac4 showing gRNA target associated with the protospacer

632 adjacent motif (5'-NGG, PAM) upstream of the Mef2c binding domain sequence. The

633 histone deacetylase domain sequence is at the 3' end of the gene. Green arrows

634 indicate forward (exon 3F) and reverse (exon 6R) primers for RT-PCR and sequencing

635 of cDNA. Red arrows indicate intronic genotyping primers (hdac4 F6, hdac4 R6)

636 flanking exon 5 . Intron $2 / 3$ and $3 / 4$ not to scale, indicated by hash marks. B: Alignment

637 of wild-type $\left(\right.$ hdac $\left.4^{+/+}\right)$with mutant cDNA sequence showing nucleotide insertions (blue)

638 in hdac4 $4^{a i k 2 / a i k 2}$ and hdac4 $4^{\text {aik3/aik3 }}$ mutants in exon 5. C and D: Insertion of nucleotides

639 results in reading frame shifts causing aberrant protein sequences (magenta in C, grey

640 boxes in D), loss of the Mef2c binding domain (indicated in yellow in C), and premature

641 termination of the protein sequence (asterisk in C indicates stop codon). E and F: RT-

642 PCR showing hdac4 cDNA is spliced correctly in mutants and there is no evidence of

643 splice variants. The wild-type cDNA product is expected to be $535 \mathrm{bp}$ and mutant bands

644 are $554 \mathrm{bp}(\mathrm{E})$ and $571 \mathrm{bp}(\mathrm{F})$. The larger band in hdac4aik3 mutants (indicated by white

645 asterisk in F) was sequenced and determined to be identical to the lower band. $G$ and

$646 \mathrm{H}$ : Genomic DNA samples were genotyped by PCR and show differences in band sizes

647 indicating mutant (841 bp hdac4aik2 mutant, 858 bp hdac4aik3 mutant), wild-type (822bp),

648 and heterozygous fish (mutant and wild-type bands). I and J: At 7dpf, mutant hdac4aik3

649 fish (J) have no apparent external abnormalities compared to wild-type siblings (I). $\mathrm{H}_{2} \mathrm{O}$

650 = negative control. $100 \mathrm{bp}$ ladder. 
652 Figure 2: Analysis of ossification of the pharyngeal skeleton of hdac4 zygotic mutants 653 stained using Alcian Blue and Alizarin Red dyes. A: Schematic showing elements of the $6547 \mathrm{dpf}$ larvae pharyngeal skeleton, lateral view. Cartilage is indicated in blue, bone is 655 indicated in red. B and C: Total scores assigned to $7 \mathrm{dpf}$ larvae scored for hdac4aik2 and

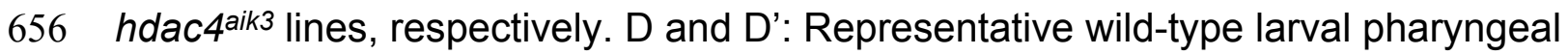
657 skeleton and enlarged view of ceratohyal showing small area (less than approximately $65820 \%$ of total area of element) of bone formation, with even borders, indicated by arrow. 659 Asterisk indicates perichondral bone collar. Lateral view. E and E': Representative $660 h^{h d a c} 4^{a i k 2}$ mutant pharyngeal skeleton and enlarged view of ceratohyal showing large 661 area (greater than approximately $20 \%$ of total area of element) of bone formation, with 662 irregular borders, indicated by arrow. Asterisks indicate perichondral bone collar. Lateral 663 view. F and G: Bar graphs comparing ratios of bone to total area of the ceratohyal for $664 h^{2} d_{a c} 4^{a i k 2}$ and hdac4 ${ }^{a i k 3}$ lines. $\mathrm{H}$ and I: Bar graphs comparing ratios of bone to total area 665 of the hyosymplectic for hdac4aik2 and hdac4aik3 lines. Bars in graph represent means 666 and error bars are standard errors of means (SEM). F: hdac4aik2: WT n=38, 667 mean $=0.102$, SEM $=0.013$; heterozygote $n=23$, mean $=0.113, \mathrm{SEM}=0.017$; and mutant $668 \mathrm{n}=32$, mean=0.145, SEM=0.0112. G: hdac4aik3: WT $\mathrm{n}=17$, mean=0.071, SEM=0.015; 669 heterozygote $n=28$, mean $=0.125$, SEM $=0.011$; and mutant $n=16$ mean $=0.151$,

670 SEM=0.016. H: hdac4aik2 : WT $n=38$, mean=0.097, SEM=0.009; heterozygote $n=23$, 671 mean=0.109, SEM=0.015; and mutant $n=32$, mean=0.133, SEM=0.011. I: hdac4 $^{\text {aik3 }}$ :

672 WT $n=17$, mean=0.142, SEM=0.008; heterozygote $n=28$, mean $=0.120, S E M=0.007$;

673 and mutant $\mathrm{n}=16$, mean $=0.151, \mathrm{SEM}=0.006$. Abbreviations: $\mathrm{ch}=$ ceratohyal, $\mathrm{bsr}=$ 
674 branchiostegal ray, de = dentary, en $=$ entopterygoid, hs $=$ hyosymplectic, ih $=$ interhyal,

$675 \mathrm{~m}=$ Meckel's cartilage,$o p=$ opercle,$p q=$ palatoquadrate,$q=$ quadrate $.{ }^{*} p \leq 0.05,{ }^{* *} p$

$676 \leq 0.01,{ }^{* * *} p \leq 0.001$. Scale bar $=100$ microns. Cartilage is stained blue (Alcian Blue),

677 bone is stained red (Alizarin Red).

678

679 Figure 3: Expression of hdac4 and sox9a mRNA in the pharyngeal skeleton of wild-type 680 embryos at $72 \mathrm{hpf}$, detected using mRNA in situ hybridization. A: Schematic of skeletal 681 elements, lateral view for B-D, blue indicates cartilage. B-D: expression of hdac4 and sox9a, arrows indicate expression in hyosymplectic, ceratohyal, and palatoquadrate

cartilages. E: Schematic of skeletal elements, ventro-lateral view for F-H. F-H:

expression of $h d a c 4$ and sox9a, arrows indicate expression in ceratohyal and indicates bone, ventral view for J-L. J-L: expression of hdac4 and sox9a, arrows indicate expression in the posterior pharyngeal arches. Abbreviations: $b b=$ basibranchial, $\mathrm{ch}=$ ceratohyal, $\mathrm{hs}=$ hyosymplectic, ih $=$ interhyal, $\mathrm{m}=$ Meckel's cartilage, op = opercle, pa3-7 = posterior pharyngeal arches 3-7, pq = palatoquadrate .

Scale bar $=50$ microns.

691

692 Figure 4: Expression of runx2a, runx2b, and sp7 mRNA in the pharyngeal skeleton of 693 wild-type and mutant larvae at 4dpf, detected using mRNA in situ hybridization using the 694 hdac4 $4^{a i k 3}$ line. A, C, E, G, I, K: Schematic views of skeletal elements, ventral view, blue 695 indicates cartilage, red indicates bone. B and D: expression of runx2a in wild-type (B) 696 and mutant (D), arrows indicate expression at the posterior margin of the ceratohyal. $F$ 
697 and $\mathrm{H}$ : expression of runx $2 b$ in wild-type $(\mathrm{F})$ and mutant $(\mathrm{H})$, arrows indicate expression

698 at the posterior margin of the ceratohyal. $\mathrm{J}$ and L: expression of $s p 7$ in wild-type $(\mathrm{J})$ and

699 mutant (L), arrows indicate expression at the posterior margin of the ceratohyal. $M$ and

$700 \mathrm{~N}$ : schematic showing overlapping domains of expression of runx2a, runx $2 b$, and sp7 in

701 wild-type $(\mathrm{M})$ and mutant $(\mathrm{N})$ larvae. $\mathrm{O}, \mathrm{P}, \mathrm{Q}$ : Average length of expression, bars in

702 graph represent means and error bars are standard errors of means (SEM); runx2a

703 (wild-type $n=10$, mean=52.9, SEM= 2.86; mutant $n=11$, mean=61.55, SEM=1.95),

704 runx2b (wild-type $n=8$, mean=30.33, SEM=1.34; mutant $n=9$, mean=35.65, SEM=0.82),

705 sp7 (wild-type $n=12$, mean=21.73, SEM= 3.65; mutant $n=10$, mean=21.78, $S E M=3.15$ ).

706 Abbreviations: $\mathrm{ch}=$ ceratohyal, $\mathrm{bsr}=$ branchiostegal ray, $\mathrm{pa} 3=$ pharyngeal $\operatorname{arch} 3, \mathrm{pq}=$

707 palatoquadrate. ${ }^{*} p \leq 0.05,{ }^{* *} p \leq 0.01$. B, D: scale bar $=50$ microns, F, H, J, L: scale

708 bar $=25$ microns. Note that anterior and posterior lengths were at measured at different

709 positions within the z-stack, and not all maximum lengths (anterior, posterior, right, and

710 left ceratohyal) are represented in the sections shown in $B, D, F$, and $\mathrm{H}$.

711

712 Figure 5: Analysis of maternal-zygote mutant and heterozygote cartilage ossification

713 using specimens from the hdac4 ${ }^{a i k 3}$ line, stained using Alcian Blue and Alizarin Red

714 dyes. A: Wild-type (non-sibling) larval pharyngeal skeleton at $7 \mathrm{dpf}$, lateral view. B: Wild-

715 type (non-sibling) larval pharyngeal skeleton at $12 \mathrm{dpf}$, lateral view. C-E: maternal-

716 zygotic mutant ( $C$ and $D$ ) and heterozygote $(E)$ pharyngeal skeletons at $7 \mathrm{dpf}$ showing

717 premature ossification of the anguloarticular bone, ventral hypohyal, and ossification of

718 the symplectic of the hyosymplectic (indicated by asterisk), lateral views. F: Total scores

719 of maternal-zygotic mutants and heterozygotes for premature/excessive ossification 
720 defects. $\mathrm{G}$ and $\mathrm{H}$ : Wild-type posterior pharyngeal arches 3 and 4 at $7 \mathrm{dpf}(\mathrm{G})$ and $12 \mathrm{dpf}$

$721(\mathrm{H})$, including ossification of first and second ceratobranchial cartilages at $12 \mathrm{dpf}$,

722 indicated by arrows, ventral views. I: Maternal-zygotic mutant showing ossification of the

723 first and second ceratobranchial cartilages cartilages at $7 \mathrm{dpf}$, indicated by arrows,

724 ventral view. Abbreviations: $a a=$ anguloarticular, $c b 1,2=$ ceratobranchial 1 and $2, q=$

725 quadrate, and hhv = ventral hypohyal. A-E: Scale bar $=200$ microns, G-I: Scale bar $=$

72650 microns. Cartilage is stained blue (Alcian Blue), bone is stained red (Alizarin Red).

727 See Figure 2A for schematic of the pharyngeal skeleton at $7 \mathrm{dpf}$.

728

729 Figure 6: Analysis of maternal-zygote mutant and heterozygote first pharyngeal arch

730 defects in fixed whole mount specimens and in specimens stained using Alcian Blue

731 and Alizarin Red dyes. A: Maternal mutant heterozygote larvae, 72hpf, lateral view. B:

732 Maternal-zygotic mutant, $72 \mathrm{hpf}$, lateral view. Arrow indicates heart edema. C: Maternal-

733 zygotic mutant pharyngeal skeleton showing loss of first pharyngeal arch elements (see

734 Figs. 2A and D for reference), $7 \mathrm{dpf}$, lateral view. D and E: Neurocrania of maternal

735 mutant zygotic heterozygote larvae showing normal patterning (D) and defects (E), 7

$736 \mathrm{dpf}$, ventral views. F: Neurocranium of maternal-zygotic mutant, $7 \mathrm{dpf}$, ventral view. G:

737 Total scores of maternal-zygotic mutants and heterozygotes for first pharyngeal arch

738 skeletal defects and neurocranium defects. Abbreviations: en = entopterygoid, $\mathrm{m}=$

739 Meckel's cartilage, $\mathrm{pq}=$ palatoquadrate, $\mathrm{q}=$ quadrate. Scale bar $=200$ microns .

740 Cartilage is stained blue (Alcian Blue), bone is stained red (Alizarin Red). 
742 Figure 7: Expression of hdac4 mRNA transcripts at different stages of embryonic and

743 larval development. A: RNA-seq mRNA levels of hdac4 transcripts from zygotic to

744 larval day 5 stage. Bars indicate numbers of hdac4 transcripts per million transcripts at

745 each stage. See http://www.ebi.ac.uk/gxa/experiments/E-ERAD-475 for Expression

746 Atlas data. B: Expression of hdac4 mRNA at 4-8 cells, dorsal view. C: Expression of

747 hdac4 mRNA at 16-32 cells, dorsal view. D: Expression of hdac4 mRNA at 256-512

748 cells, dorsal view. E: Expression of hdac4 mRNA at 75-90\% epiboly, arrow indicates

749 embryo dorsal to the yolk sac, lateral view. B-D: Scale bar $=200$ microns, E: Scale bar

$750=50$ microns.

751

752

753

754 


\section{References}

756

757 Arenas-Rodríguez A, Rubiano Vargas JF, Hoyos JM. 2018. Comparative description

758

759

760

761

762

763

764

765

766

767

768

769

770

771

772

773

774

775

776

777 and ossification patterns of Dendropsophus labialis (Peters, 1863) and Scinax ruber (Laurenti, 1758) (Anura: Hylidae). PeerJ 6:e4525. DOI: 10.7717/peerj.4525.

Arnold MA, Kim Y, Czubryt MP, Phan D, McAnally J, Qi X, Shelton JM, Richardson JA, Bassel-Duby R, Olson EN. 2007. MEF2C transcription factor controls chondrocyte hypertrophy and bone development. Developmental Cell 12:377389. DOI: 10.1016/j.devcel.2007.02.004.

Avaron F, Hoffman L, Guay D, Akimenko MA. 2006. Characterization of two new zebrafish members of the hedgehog family: Atypical expression of a zebrafish indian hedgehog gene in skeletal elements of both endochondral and dermal origins. Developmental Dynamics 235:478-489. DOI: 10.1002/dvdy.20619.

Bassett AR, Tibbit C, Ponting CP, Liu J-L. 2013. Highly Efficient Targeted Mutagenesis of Drosophila with the CRISPR/Cas9 System. Cell Reports 4:220-228. DOI: 10.1016/j.celrep.2013.06.020.

Boer EF, Howell ED, Schilling TF, Jette CA, Stewart RA. 2015. Fascin1-dependent Filopodia are required for directional migration of a subset of neural crest cells. PLoS genetics 11:e1004946. DOI: 10.1371/journal.pgen.1004946.

Boer EF, Jette CA, Stewart RA. 2016. Neural Crest Migration and Survival Are Susceptible to Morpholino-Induced Artifacts. PloS One 11:e0167278. DOI: 10.1371/journal.pone.0167278. 
778 Cubbage CC, Mabee PM. 1996. Development of the cranium and paired fins in the 779 zebrafish Danio rerio (Ostariophysi, Cyprinidae). Journal of Morphology 229:121160. DOI: 10.1002/(SICI)1097-4687(199608)229:2<121::AID-JMOR1>3.0.CO;2-

782 DeLaurier A, Eames BF, Blanco-Sánchez B, Peng G, He X, Swartz ME, Ullmann B, 783 Westerfield M, Kimmel CB. 2010. Zebrafish sp7:EGFP: a transgenic for studying otic vesicle formation, skeletogenesis, and bone regeneration. Genesis (New York, N.Y.: 2000) 48:505-511. DOI: 10.1002/dvg.20639.

DeLaurier A, Nakamura Y, Braasch I, Khanna V, Kato H, Wakitani S, Postlethwait JH, Kimmel CB. 2012. Histone deacetylase-4 is required during early cranial neural crest development for generation of the zebrafish palatal skeleton. $B M C$ developmental biology 12:16. DOI: 10.1186/1471-213X-12-16.

Eames BF, DeLaurier A, Ullmann B, Huycke TR, Nichols JT, Dowd J, McFadden M, Sasaki MM, Kimmel CB. 2013. FishFace: interactive atlas of zebrafish craniofacial development at cellular resolution. BMC developmental biology 13:23. DOI: 10.1186/1471-213X-13-23.

Eames BF, Yan Y-L, Swartz ME, Levic DS, Knapik EW, Postlethwait JH, Kimmel CB. 2011. Mutations in fam $20 \mathrm{~b}$ and xylt 1 reveal that cartilage matrix controls timing of endochondral ossification by inhibiting chondrocyte maturation. PLoS genetics 7:e1002246. DOI: 10.1371/journal.pgen.1002246.

Flores MV, Tsang VWK, Hu W, Kalev-Zylinska M, Postlethwait J, Crosier P, Crosier K, Fisher S. 2004. Duplicate zebrafish runx2 orthologues are expressed in 
developing skeletal elements. Gene Expression Patterns 4:573-581. DOI:

801 10.1016/j.modgep.2004.01.016.

802 Gao B, Guo J, She C, Shu A, Yang M, Tan Z, Yang X, Guo S, Feng G, He L. 2001.

803 Mutations in $\mathrm{IHH}$, encoding Indian hedgehog, cause brachydactyly type A-1. Nature Genetics 28:386-388. DOI: 10.1038/ng577.

805 Grünbaum T, Cloutier R, Vincent B. 2012. Dynamic skeletogenesis in fishes: insight of 806 exercise training on developmental plasticity. Developmental Dynamics: An Official Publication of the American Association of Anatomists 241:1507-1524. DOI: $10.1002 / d v d y .23837$.

Haberland M, Montgomery RL, Olson EN. 2009. The many roles of histone deacetylases in development and physiology: implications for disease and therapy. Nature Reviews. Genetics 10:32-42. DOI: 10.1038/nrg2485.

812 Hall BK. 2014. Bones and Cartilage: Developmental and Evolutionary Skeletal Biology. $813 \quad$ Academic Press.

814 Hammond CL, Schulte-Merker S. 2009. Two populations of endochondral osteoblasts

815 with differential sensitivity to Hedgehog signalling. Development (Cambridge,

816 England) 136:3991-4000. DOI: 10.1242/dev.042150.

817 Harrington SM, Harrison LB, Sheil CA. 2013. Ossification sequence heterochrony 818 among amphibians. Evolution \& Development 15:344-364. DOI:

$819 \quad 10.1111 /$ ede.12043.

820 Huycke TR, Eames BF, Kimmel CB. 2012. Hedgehog-dependent proliferation drives

821 modular growth during morphogenesis of a dermal bone. Development

822 (Cambridge, England) 139:2371-2380. DOI: 10.1242/dev.079806. 
823 Hwang WY, Fu Y, Reyon D, Maeder ML, Tsai SQ, Sander JD, Peterson RT, Yeh J-RJ,

824 Joung JK. 2013. Efficient genome editing in zebrafish using a CRISPR-Cas

825 system. Nature Biotechnology 31:227-229. DOI: 10.1038/nbt.2501.

826 Karsenty G, Wagner EF. 2002. Reaching a genetic and molecular understanding of $827 \quad$ skeletal development. Developmental Cell 2:389-406.

828 Kearse M, Moir R, Wilson A, Stones-Havas S, Cheung M, Sturrock S, Buxton S, Cooper 829 A, Markowitz S, Duran C, Thierer T, Ashton B, Meintjes P, Drummond A. 2012.

830 Geneious Basic: an integrated and extendable desktop software platform for the 831 organization and analysis of sequence data. Bioinformatics (Oxford, England) 28:1647-1649. DOI: 10.1093/bioinformatics/bts199.

833 Kimmel CB, Ballard WW, Kimmel SR, Ullmann B, Schilling TF. 1995. Stages of 834 embryonic development of the zebrafish. Developmental Dynamics: An Official 835 Publication of the American Association of Anatomists 203:253-310. DOI: 10.1002/aja.1002030302.

837 Komori T, Yagi H, Nomura S, Yamaguchi A, Sasaki K, Deguchi K, Shimizu Y, Bronson 838 RT, Gao YH, Inada M, Sato M, Okamoto R, Kitamura Y, Yoshiki S, Kishimoto T. 839 1997. Targeted disruption of Cbfa1 results in a complete lack of bone formation $840 \quad$ owing to maturational arrest of osteoblasts. Cell 89:755-764.

841 Labun K, Montague TG, Gagnon JA, Thyme SB, Valen E. 2016. CHOPCHOP v2: a web 842 tool for the next generation of CRISPR genome engineering. Nucleic Acids $843 \quad$ Research 44:W272-W276. DOI: 10.1093/nar/gkw398.

844 Li N, Felber K, Elks P, Croucher P, Roehl HH. 2009. Tracking gene expression during 845 zebrafish osteoblast differentiation. Developmental Dynamics: An Official 
Publication of the American Association of Anatomists 238:459-466. DOI:

847 $10.1002 / d v d y .21838$.

848 Lu J, McKinsey TA, Nicol RL, Olson EN. 2000. Signal-dependent activation of the MEF2 849 transcription factor by dissociation from histone deacetylases. Proceedings of the National Academy of Sciences of the United States of America 97:4070-4075. DOI: 10.1073/pnas.080064097.

Maeda Y, Nakamura E, Nguyen M-T, Suva LJ, Swain FL, Razzaque MS, Mackem S, Lanske B. 2007. Indian Hedgehog produced by postnatal chondrocytes is essential for maintaining a growth plate and trabecular bone. Proceedings of the National Academy of Sciences of the United States of America 104:6382-6387. DOI: $10.1073 /$ pnas.0608449104.

McKinsey TA, Zhang CL, Lu J, Olson EN. 2000. Signal-dependent nuclear export of a histone deacetylase regulates muscle differentiation. Nature 408:106-111. DOI: $10.1038 / 35040593$.

van der Meulen T, Kranenbarg S, Schipper H, Samallo J, van Leeuwen JL, Franssen H. 2005. Identification and characterisation of two runx2 homologues in zebrafish with different expression patterns. Biochimica Et Biophysica Acta 1729:105-117. DOI: 10.1016/j.bbaexp.2005.03.008.

864 Montague TG, Cruz JM, Gagnon JA, Church GM, Valen E. 2014. CHOPCHOP: a CRISPR/Cas9 and TALEN web tool for genome editing. Nucleic Acids Research 42:W401-W407. DOI: 10.1093/nar/gku410.

Nagata M, Nuckolls GH, Wang X, Shum L, Seki Y, Kawase T, Takahashi K, Nonaka K, Takahashi I, Noman AA, Suzuki K, Slavkin HC. 2011. The primary site of the 
acrocephalic feature in Apert Syndrome is a dwarf cranial base with accelerated chondrocytic differentiation due to aberrant activation of the FGFR2 signaling. Bone 48:847-856. DOI: 10.1016/j.bone.2010.11.014.

872 Nakashima K, Zhou X, Kunkel G, Zhang Z, Deng JM, Behringer RR, de Crombrugghe B. 2002. The novel zinc finger-containing transcription factor osterix is required for osteoblast differentiation and bone formation. Cell 108:17-29.

875 Nishio Y, Dong Y, Paris M, O’Keefe RJ, Schwarz EM, Drissi H. 2006. Runx2-mediated regulation of the zinc finger Osterix/Sp7 gene. Gene 372:62-70. DOI: 10.1016/j.gene.2005.12.022.

Passier R, Zeng H, Frey N, Naya FJ, Nicol RL, McKinsey TA, Overbeek P, Richardson JA, Grant SR, Olson EN. 2000. CaM kinase signaling induces cardiac hypertrophy and activates the MEF2 transcription factor in vivo. The Journal of Clinical Investigation 105:1395-1406. DOI: 10.1172/JCI8551.

Quack I, Vonderstrass B, Stock M, Aylsworth AS, Becker A, Brueton L, Lee PJ, Majewski F, Mulliken JB, Suri M, Zenker M, Mundlos S, Otto F. 1999. Mutation analysis of core binding factor A1 in patients with cleidocranial dysplasia. American Journal of Human Genetics 65:1268-1278.

Rothschild SC, Lee HJ, Ingram SR, Mohammadi DK, Walsh GS, Tombes RM. 2018. Calcium signals act through histone deacetylase to mediate pronephric kidney morphogenesis. Developmental Dynamics: An Official Publication of the American Association of Anatomists 247:807-817. DOI: 10.1002/dvdy.24632. 
890 Schneider CA, Rasband WS, Eliceiri KW. 2012.NIH Image to Image J: 25 years of $891 \quad$ image analysis. Available at $h t t p s: / / w w w . n a t u r e . c o m / a r t i c l e s / n m e t h .2089$ 892 (accessed October 3, 2018). DOI: 10.1038/nmeth.2089.

893 Sharpe JJ, Cooper TA. 2017. Unexpected consequences: exon skipping caused by 894 CRISPR-generated mutations. Genome Biology 18:109. DOI: 10.1186/s13059$895 \quad 017-1240-0$.

896 Tadros W, Lipshitz HD. 2009. The maternal-to-zygotic transition: a play in two acts. 897 Development 136:3033-3042. DOI: 10.1242/dev.033183.

898 Talbot JC, Johnson SL, Kimmel CB. 2010. hand2 and Dlx genes specify dorsal, 899 intermediate and ventral domains within zebrafish pharyngeal arches.

900 Development (Cambridge, England) 137:2507-2517. DOI: 10.1242/dev.049700.

901 Thisse B, Thisse C. 2014. In situ hybridization on whole-mount zebrafish embryos and 902 young larvae. Methods in Molecular Biology (Clifton, N.J.) 1211:53-67. DOI: 903 10.1007/978-1-4939-1459-3_5.

904 Truett GE, Heeger P, Mynatt RL, Truett AA, Walker JA, Warman ML. 2000. Preparation 905 of PCR-quality mouse genomic DNA with hot sodium hydroxide and tris 906 (HotSHOT). BioTechniques 29:52, 54.

907 Valadares ER, Carneiro TB, Santos PM, Oliveira AC, Zabel B. 2014. What is new in $908 \quad$ genetics and osteogenesis imperfecta classification? Jornal De Pediatria 90:536$909 \quad$ 541. DOI: $10.1016 / j . j p e d .2014 .05 .003$.

910 Vega RB, Matsuda K, Oh J, Barbosa AC, Yang X, Meadows E, McAnally J, Pomajzl C, 911 Shelton JM, Richardson JA, Karsenty G, Olson EN. 2004. Histone deacetylase 4 
912

913

914

915

916

\section{7}

918

919

920

921

922

923

924

925

926

927

928

929

930

controls chondrocyte hypertrophy during skeletogenesis. Cell 119:555-566. DOI: 10.1016/j.cell.2004.10.024.

van der Velden YU, Wang L, Querol Cano L, Haramis A-PG. 2013. The polycomb group protein ring $1 \mathrm{~b} / \mathrm{rnf} 2$ is specifically required for craniofacial development. PloS One 8:e73997. DOI: 10.1371/journal.pone.0073997.

Vortkamp A, Lee K, Lanske B, Segre GV, Kronenberg HM, Tabin CJ. 1996. Regulation of rate of cartilage differentiation by Indian hedgehog and PTH-related protein. Science (New York, N.Y.) 273:613-622.

Walker MB, Kimmel CB. 2007. A two-color acid-free cartilage and bone stain for zebrafish larvae. Biotechnic \& Histochemistry: Official Publication of the Biological Stain Commission 82:23-28. DOI: 10.1080/10520290701333558.

Wasden MB, Roberts RL, DeLaurier A. 2017. Optimizing Sperm Collection Procedures in Zebrafish. Journal of the South Carolina Academy of Science 15.

Wysolmerski JJ, Cormier S, Philbrick WM, Dann P, Zhang JP, Roume J, Delezoide AL, Silve C. 2001. Absence of functional type 1 parathyroid hormone (PTH)/PTHrelated protein receptors in humans is associated with abnormal breast development and tooth impaction. The Journal of Clinical Endocrinology and Metabolism 86:1788-1794. DOI: 10.1210/jcem.86.4.7404. 


\section{Figure 1}

Overview of CRISPR strategy and generation of hdac4 mutant lines.

A: Genomic structure of hdac4 showing gRNA target associated with the protospacer adjacent motif (5'-NGG, PAM) upstream of the Mef2c binding domain sequence. The histone deacetylase domain sequence is at the $3^{\prime}$ end of the gene. Green arrows indicate forward (exon $3 F$ ) and reverse (exon 6R) primers for RT-PCR and sequencing of cDNA. Red arrows indicate intronic genotyping primers (hdac4 F6, hdac4 R6) flanking exon 5. Intron 2/3 and 3/4 not to scale, indicated by hash marks. B: Alignment of wild-type $\left(\right.$ hdac $\left.^{+/+}\right)$with mutant cDNA sequence showing nucleotide insertions (blue) in hdac4 $4^{\text {aikz/aik2 }}$ and hdac4 $4^{\text {aik3/aik3 }}$ mutants in exon 5. C and D: Insertion of nucleotides results in reading frame shifts causing aberrant protein sequences (magenta in $C$, grey boxes in D), loss of the Mef2c binding domain (indicated in yellow in C), and premature termination of the protein sequence (asterisk in C indicates stop codon). E and F: RT-PCR showing hdac4 CDNA is spliced correctly in mutants and there is no evidence of splice variants. The wild-type cDNA product is expected to be $535 \mathrm{bp}$ and mutant bands are $554 \mathrm{bp}(\mathrm{E})$ and $571 \mathrm{bp}(\mathrm{F})$. The larger band in hdac4 ${ }^{a i k 3}$ mutants (indicated by white asterisk in F) was sequenced and determined to be identical to the lower band. $\mathrm{G}$ and $\mathrm{H}$ : Genomic DNA samples were genotyped by PCR and show differences in band sizes indicating mutant (841 bp hdac4 ${ }^{\text {aik2 }}$ mutant, 858 bp hdac4 $4^{\text {aik3 }}$ mutant), wild-type (822bp), and heterozygous fish (mutant and wild-type bands). I and J: At 7dpf, mutant hdac4 ${ }^{a i k 3}$ fish (J) have no apparent external abnormalities compared to wild-type siblings (I). $\mathrm{H}_{2} \mathrm{O}=$ negative control. 100 bp ladder. 


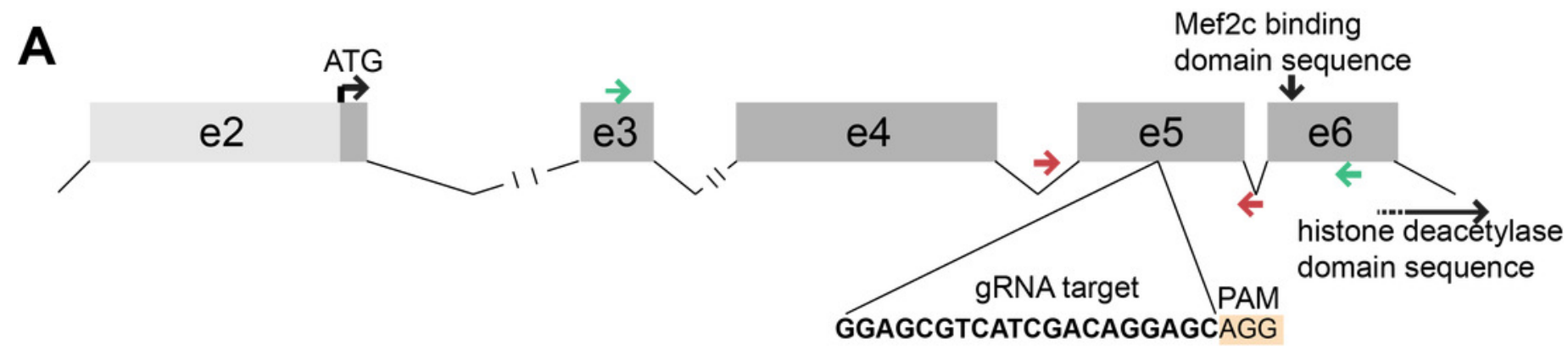

B hdac4 $4^{+/+}$

CGTCATCGACAGG---------------------AGCAGGAGATGGAGAAACAGCAGCGAG

hdac4 aikzaik2 CGTCATCGACAGGAGATGATCGACAGGAGCATAGCAGGAGATGGAGAAACAGCAGCGAG

hdac4 ${ }^{+/+} \quad$ CGTCATCG--ACAGGAG---------------------------CAGGAGATGGAGAAA

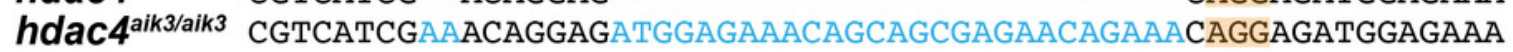

C

exon $5 / 6$ mef2c binding splice domain

Hdac4 WT HQQDLLALKHQQELLEHQRKLERHRQEQEMEKQQREQKLQLLKNKERGQESAVASTEVKMRLQEFVLN

Hdac4 aik2 HQQDLLALKHQQELLEHQRKLERHRQEMIDRSIAGDGETAARTEATVTQKQRARTRERRGEH*

Hdac4 aik3 HQQDLLALKHQQELLEHQRKLERHRNRRWRNSSENRNRRWRNSSENRSYSYSKTKSADKRAPWRALRSR*

D

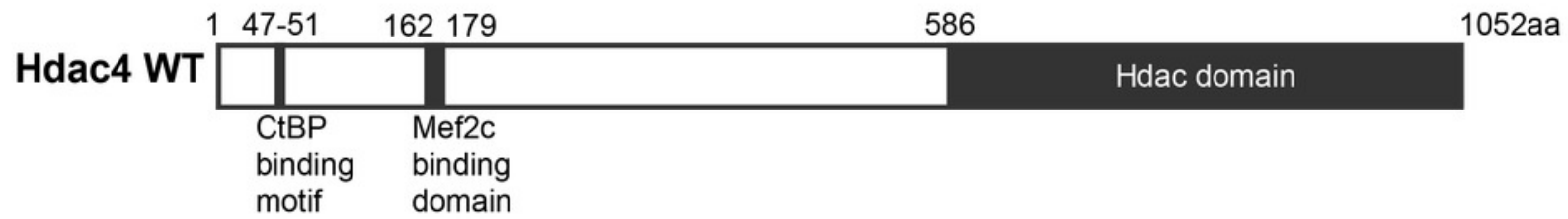

Hdac4 aik2

Hdac4 aik3

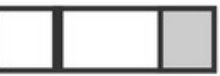

174aa

$181 \mathrm{aa}$
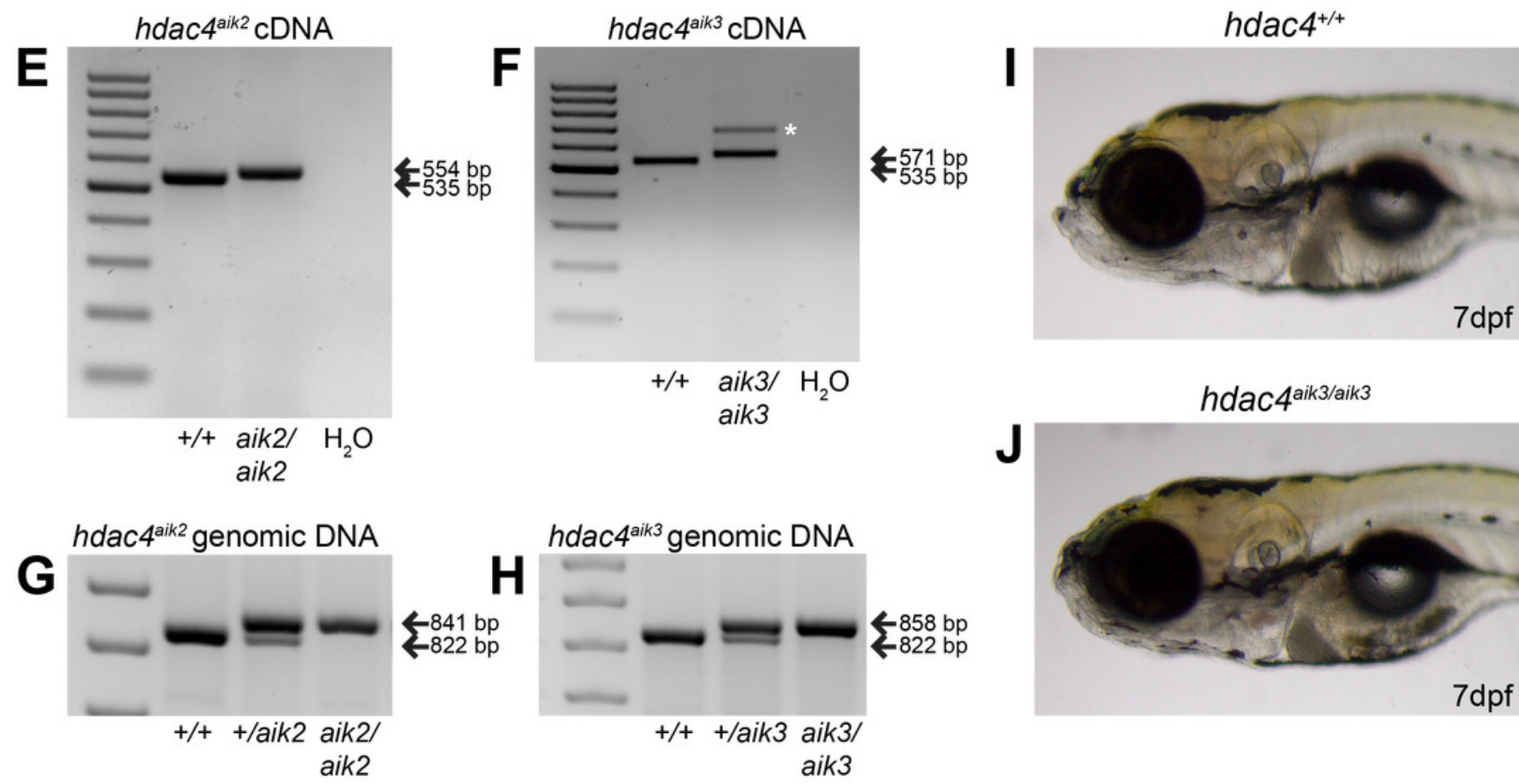

hdac4 $^{\text {aik3 }}$ genomic DNA

4

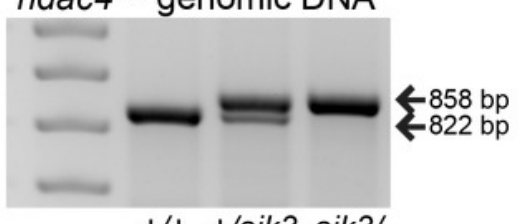

+/+ +/aik3 aik3/

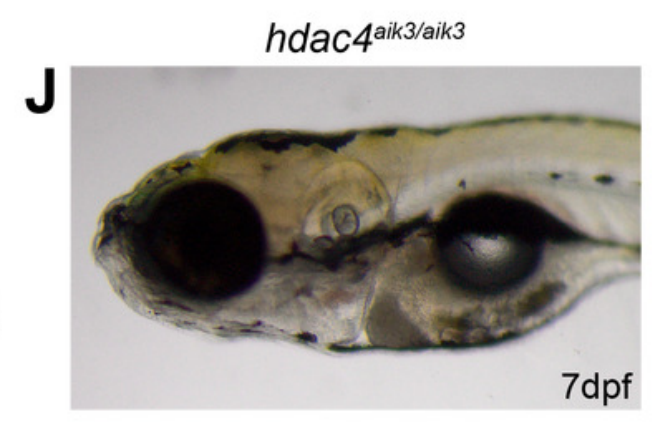




\section{Figure 2}

Analysis of ossification of the pharyngeal skeleton of hdac4 zygotic mutants stained using Alcian Blue and Alizarin Red dyes.

A: Schematic showing elements of the $7 \mathrm{dpf}$ larvae pharyngeal skeleton, lateral view.

Cartilage is indicated in blue, bone is indicated in red. B and C: Total scores assigned to $7 \mathrm{dpf}$ larvae scored for $h d a c 4^{a i k 2}$ and $h d a c 4^{a i k 3}$ lines, respectively. D and D': Representative wildtype larval pharyngeal skeleton and enlarged view of ceratohyal showing small area (less than approximately $20 \%$ of total area of element) of bone formation, with even borders, indicated by arrow. Asterisk indicates perichondral bone collar. Lateral view. E and E': Representative hdac4 ${ }^{\text {aik2 }}$ mutant pharyngeal skeleton and enlarged view of ceratohyal showing large area (greater than approximately $20 \%$ of total area of element) of bone formation, with irregular borders, indicated by arrow. Asterisks indicate perichondral bone collar. Lateral view. F and G: Bar graphs comparing ratios of bone to total area of the ceratohyal for hdac4 $4^{\text {aik2 }}$ and $h d a c 4^{\text {aik3 }}$ lines. $\mathrm{H}$ and I: Bar graphs comparing ratios of bone to total area of the hyosymplectic for $h d a c 4^{a i k 2}$ and $h d a c 4^{\text {aik3 }}$ lines. Bars in graph represent means and error bars are standard errors of means (SEM). F: hdac4aikz: WT $n=38$, mean $=0.102$, $S E M=0.013$; heterozygote $n=23$, mean $=0.113$, $S E M=0.017$; and mutant $n=32$, mean $=0.145, S E M=0.0112$. G: hdac4 $^{\text {aik3 }}:$ WT $n=17$, mean $=0.071, S E M=0.015$; heterozygote $n=28$, mean $=0.125$, SEM $=0.011$; and mutant $n=16$ mean $=0.151, S E M=0.016$. $\mathrm{H}$ : hdac4 ${ }^{\text {aik2}}$ : WT $\mathrm{n}=38$, mean $=0.097, \mathrm{SEM}=0.009$; heterozygote $\mathrm{n}=23$, mean $=0.109$, $\mathrm{SEM}=0.015$; and mutant $\mathrm{n}=32$, mean $=0.133$, SEM $=0.011$. I: hdac4 ${ }^{\text {aik3 }}: \mathrm{WT} n=17$, mean $=0.142$, SEM $=0.008$; heterozygote $n=28$, mean $=0.120, S E M=0.007$; and mutant $n=16$, mean $=0.151, \mathrm{SEM}=0.006$. Abbreviations: $\mathrm{ch}=$ ceratohyal, $\mathrm{bsr}=$ branchiostegal ray, $\mathrm{de}=$ dentary, en = entopterygoid, hs = hyosymplectic, in = interhyal, $\mathrm{m}=$ Meckel's cartilage, op $=$ opercle, $\mathrm{pq}=$ palatoquadrate $\mathrm{q}=$ quadrate. $* p \leq 0.05, * * p \leq 0.01, * * * \leq 0.001$. Scale bar $=100$ microns. Cartilage is stained blue (Alcian Blue), bone is stained red (Alizarin Red). 

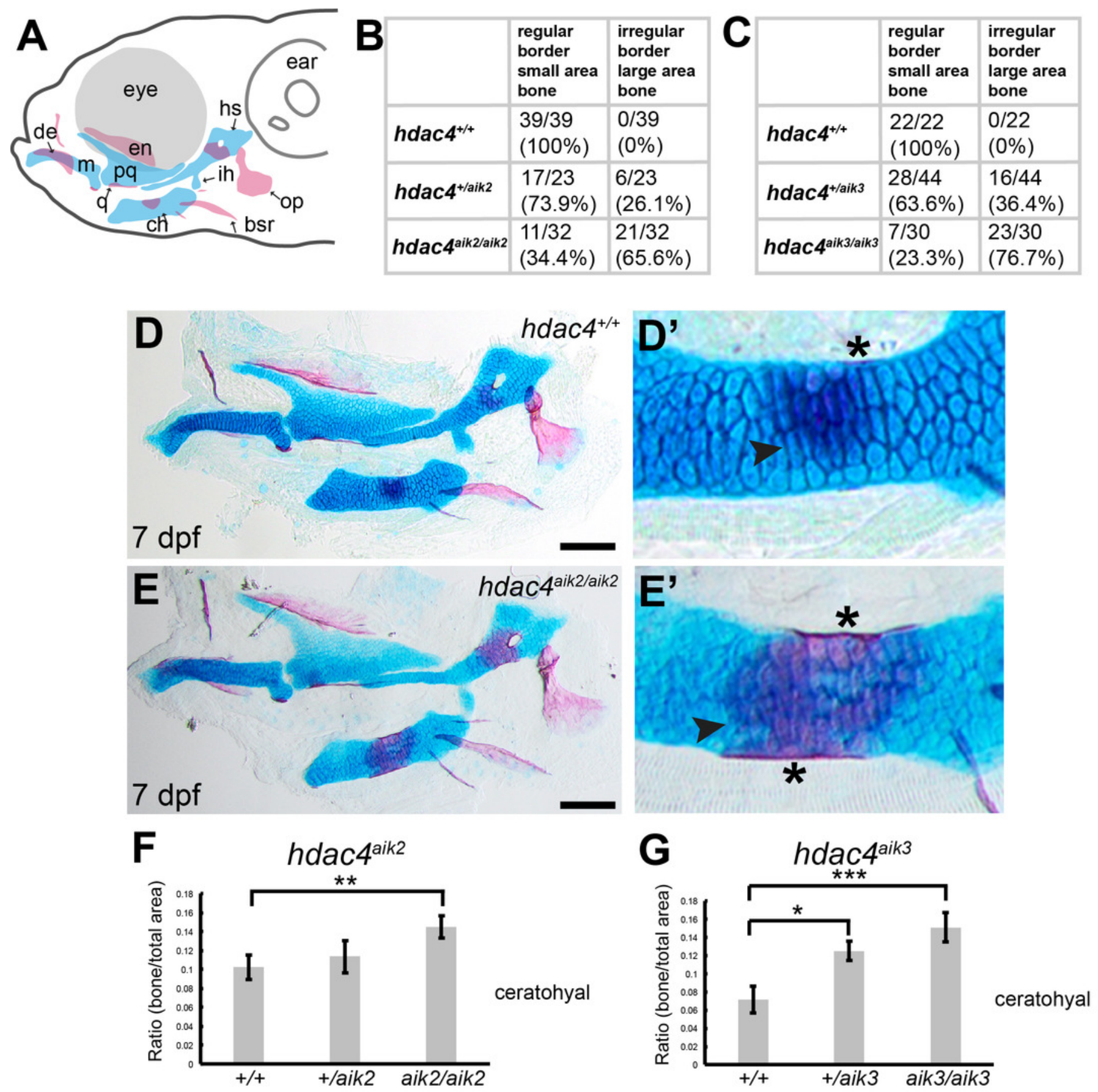

H
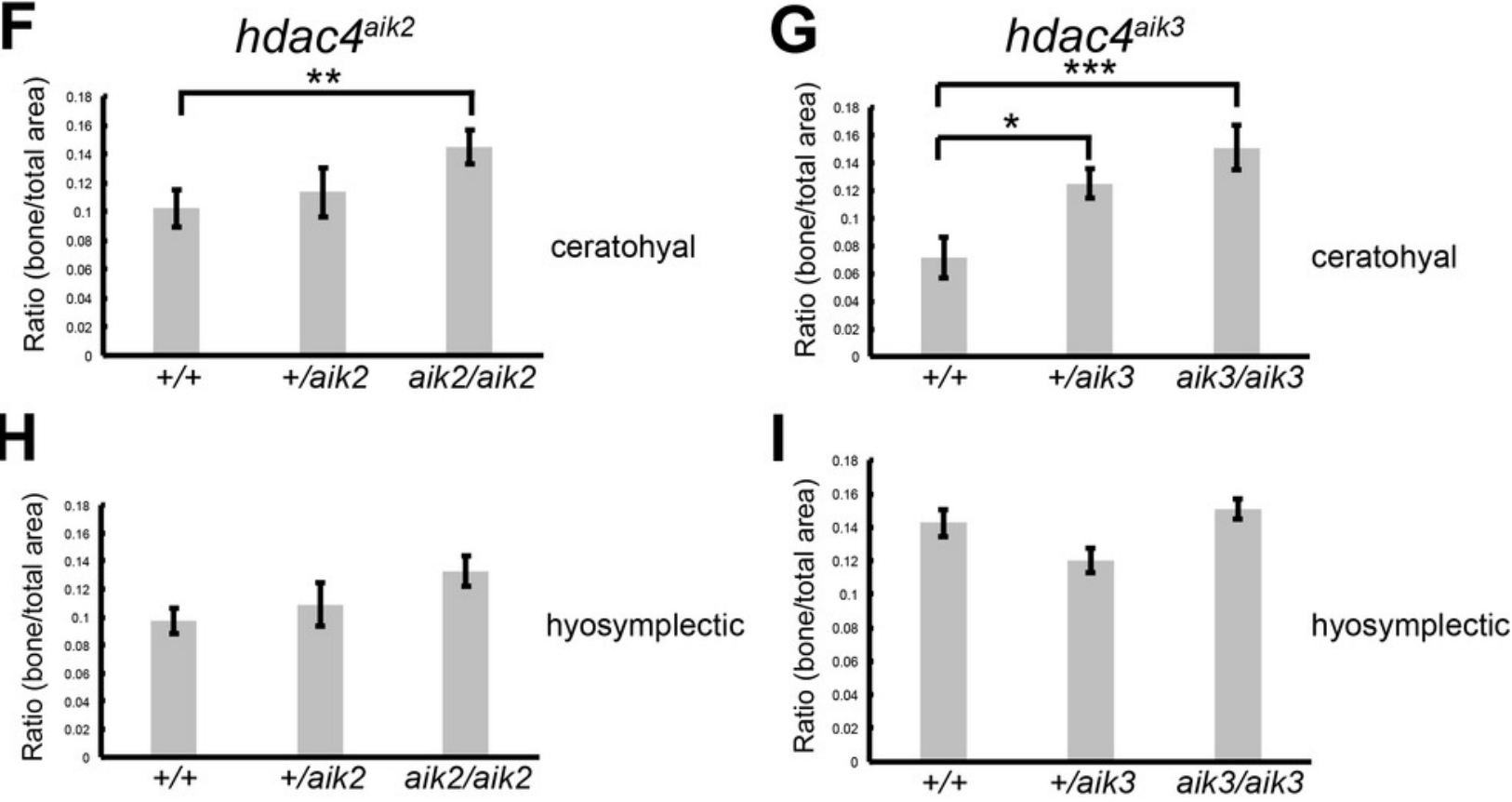


\section{Figure 3}

Expression of hdac4 and sox9a mRNA in the pharyngeal skeleton of wild-type embryos at $72 \mathrm{hpf}$, detected using mRNA in situ hybridization.

A: Schematic of skeletal elements, lateral view for B-D, blue indicates cartilage. B-D:

expression of hdac4 and sox9a, arrows indicate expression in hyosymplectic, ceratohyal, and palatoquadrate cartilages. E: Schematic of skeletal elements, ventro-lateral view for F-H. F-H: expression of hdac4 and sox9a, arrows indicate expression in ceratohyal and hyosymplectic cartilages. I: Schematic of skeletal elements, blue indicates cartilage, red indicates bone, ventral view for J-L. J-L: expression of hdac4 and sox9a, arrows indicate expression in the posterior pharyngeal arches. Abbreviations: $\mathrm{bb}=$ basibranchial, $\mathrm{ch}=$ ceratohyal, $\mathrm{hs}=$ hyosymplectic, ih = interhyal, $\mathrm{m}=$ Meckel's cartilage, $\mathrm{op}=$ opercle, $\mathrm{pa3}-7=$ posterior pharyngeal arches $3-7, \mathrm{pq}=$ palatoquadrate. Scale bar $=50$ microns. 


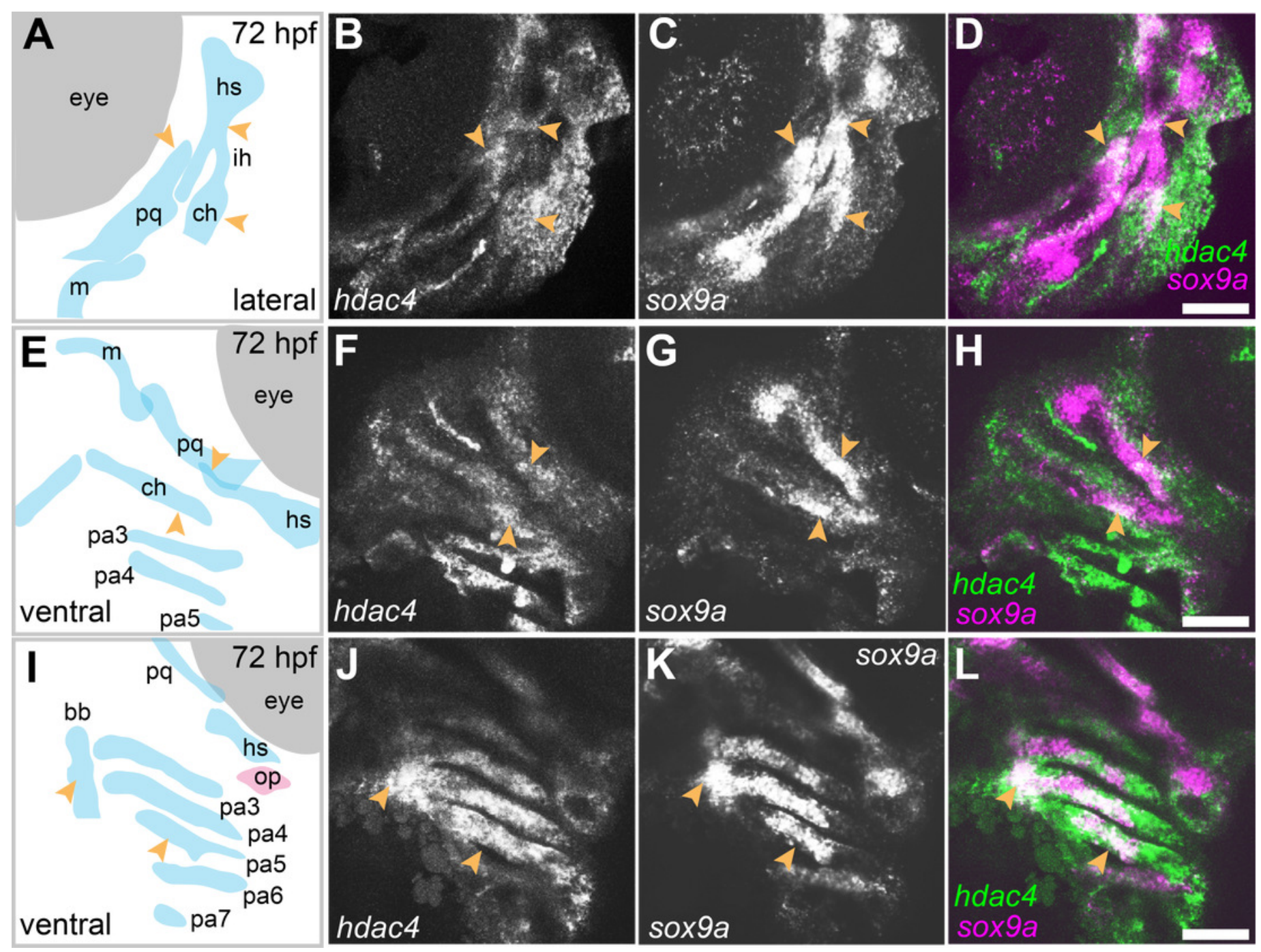




\section{Figure 4}

Expression of run $x 2 a$, run $x 2 b$, and $s p 7$ mRNA in the pharyngeal skeleton of wild-type and mutant larvae at $4 \mathrm{dpf}$, detected using mRNA in situ hybridization using the hdac4 ${ }^{\text {aik3 }}$ line.

A, C, E, G, I, K: Schematic views of skeletal elements, ventral view, blue indicates cartilage, red indicates bone. B and D: expression of runx2a in wild-type (B) and mutant (D), arrows indicate expression at the posterior margin of the ceratohyal. $\mathrm{F}$ and $\mathrm{H}$ : expression of runx $2 \mathrm{~b}$ in wild-type $(\mathrm{F})$ and mutant $(\mathrm{H})$, arrows indicate expression at the posterior margin of the ceratohyal. J and L: expression of sp7 in wild-type (J) and mutant (L), arrows indicate expression at the posterior margin of the ceratohyal. $\mathrm{M}$ and $\mathrm{N}$ : schematic showing overlapping domains of expression of runx2a, runx $2 b$, and sp7 in wild-type (M) and mutant (N) larvae. O, P, Q: Average length of expression, bars in graph represent means and error bars are standard errors of means (SEM); runx2a (wild-type $n=10$, mean $=52.9, \mathrm{SEM}=2.86$; mutant $n=11$, mean=61.55, SEM=1.95), runx2b (wild-type $n=8$, mean $=30.33, S E M=1.34$; mutant $n=9$, mean=35.65, SEM=0.82), $s p 7$ (wild-type $n=12$, mean=21.73, SEM= 3.65; mutant $\mathrm{n}=10$, mean $=21.78, \mathrm{SEM}=3.15)$. Abbreviations: $\mathrm{ch}=$ ceratohyal, $\mathrm{bsr}=$ branchiostegal ray, pa3 $=$ pharyngeal $\operatorname{arch} 3, p q=$ palatoquadrate. $* p \leq 0.05, * * p \leq 0.01$. B, D: scale bar $=50$ microns, F, H, J, L: scale bar $=25$ microns. Note that anterior and posterior lengths were at measured at different positions within the z-stack, and not all maximum lengths (anterior, posterior, right, and left ceratohyal) are represented in the sections shown in $B, D, F$, and $H$. 

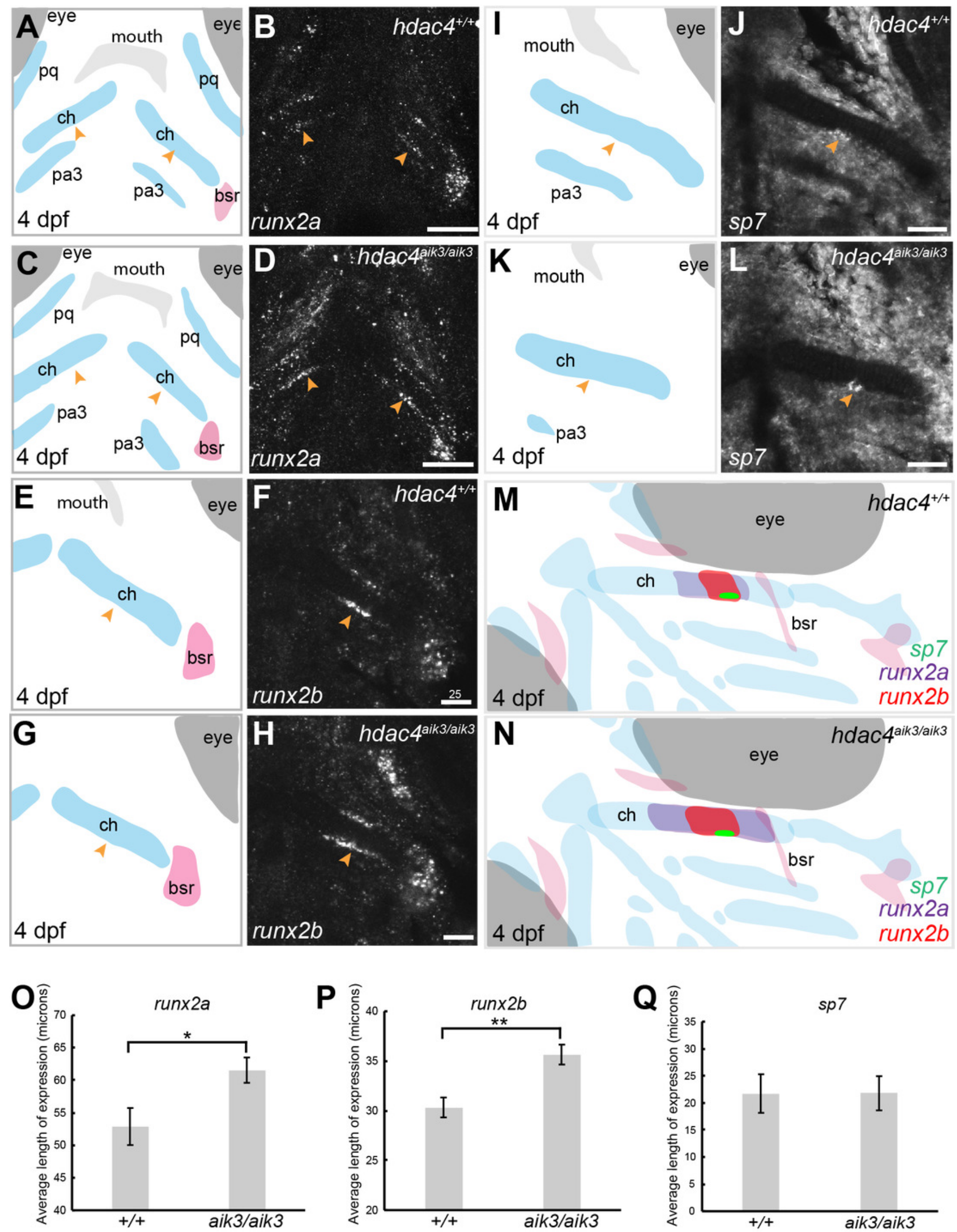


\section{Figure 5}

Analysis of maternal-zygote mutant and heterozygote cartilage ossification using specimens from the hdac4 ${ }^{\text {aik3 }}$ line, stained using Alcian Blue and Alizarin Red dyes.

A: Wild-type (non-sibling) larval pharyngeal skeleton at $7 \mathrm{dpf}$, lateral view. B: Wild-type (nonsibling) larval pharyngeal skeleton at $12 \mathrm{dpf}$, lateral view. C-E: maternal-zygotic mutant (C and D) and heterozygote (E) pharyngeal skeletons at $7 \mathrm{dpf}$ showing premature ossification of the anguloarticular bone, ventral hypohyal, and ossification of the symplectic of the hyosymplectic (indicated by asterisk), lateral views. F: Total scores of maternal-zygotic mutants and heterozygotes for premature/excessive ossification defects. G and H: Wild-type posterior pharyngeal arches 3 and 4 at $7 \mathrm{dpf}(\mathrm{G})$ and $12 \mathrm{dpf}(\mathrm{H})$, including ossification of first and second ceratobranchial cartilages at $12 \mathrm{dpf}$, indicated by arrows, ventral views. I: Maternal-zygotic mutant showing ossification of the first and second ceratobranchial cartilages cartilages at 7dpf, indicated by arrows, ventral view. Abbreviations: $a a=$ anguloarticular, cb1,2 = ceratobranchial 1 and 2, q = quadrate, and hhv = ventral hypohyal. A-E: Scale bar $=200$ microns, G-I: Scale bar $=50$ microns. Cartilage is stained blue (Alcian Blue), bone is stained red (Alizarin Red). See Figure 2A for schematic of the pharyngeal skeleton at $7 \mathrm{dpf}$. 


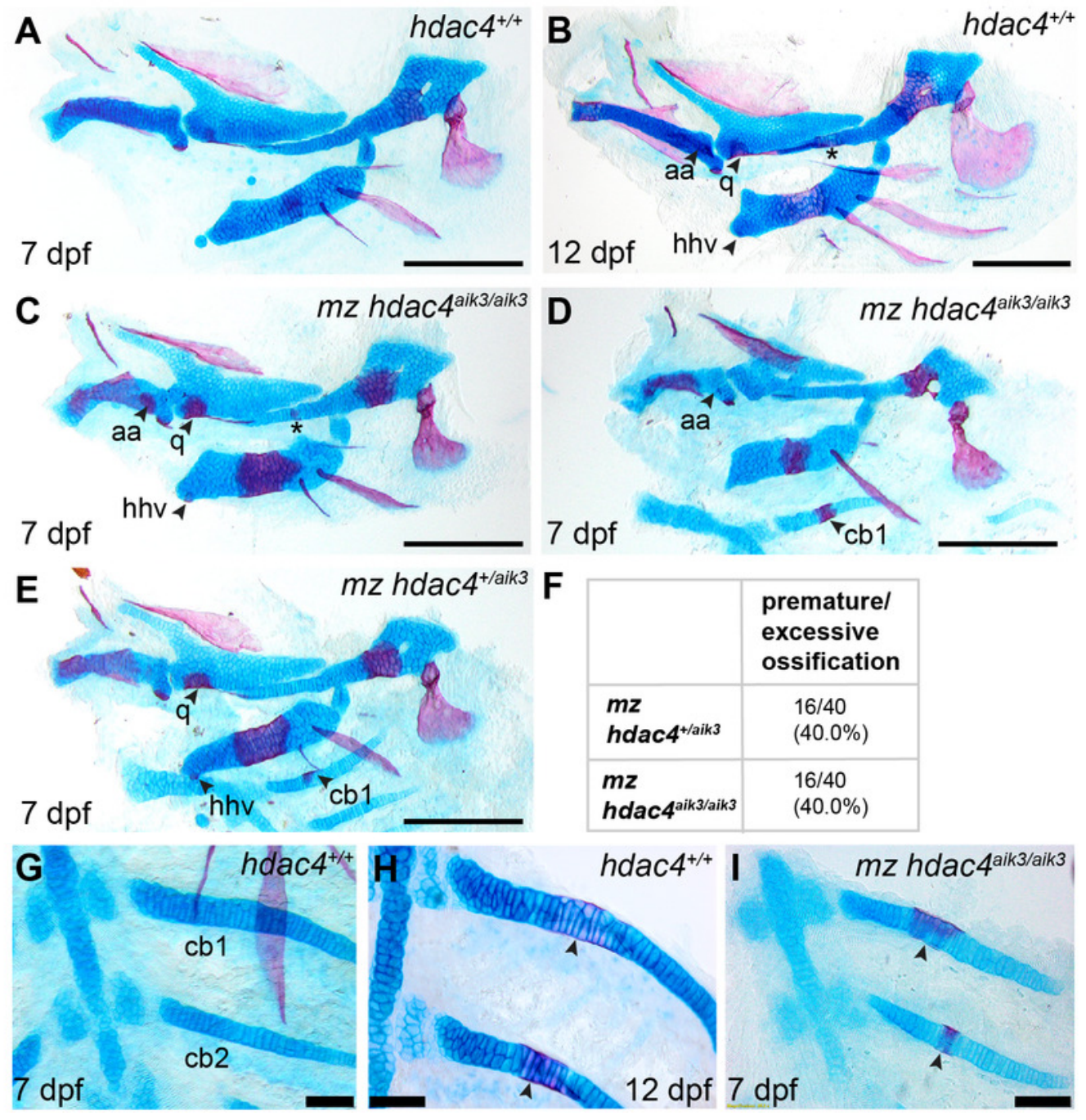




\section{Figure 6}

Analysis of maternal-zygote mutant and heterozygote first pharyngeal arch defects in fixed whole mount specimens and in specimens stained using Alcian Blue and Alizarin Red dyes.

A: Maternal mutant heterozygote larvae, $72 \mathrm{hpf}$, lateral view. B: Maternal-zygotic mutant, $72 \mathrm{hpf}$, lateral view. Arrow indicates heart edema. C: Maternal-zygotic mutant pharyngeal skeleton showing loss of first pharyngeal arch elements (see Figs. 2A and D for reference), 7 dpf, lateral view. D and E: Neurocrania of maternal mutant zygotic heterozygote larvae showing normal patterning (D) and defects (E), $7 \mathrm{dpf}$, ventral views. F: Neurocranium of maternal-zygotic mutant, $7 \mathrm{dpf}$, ventral view. G: Total scores of maternal-zygotic mutants and heterozygotes for first pharyngeal arch skeletal defects and neurocranium defects. Abbreviations: en = entopterygoid, $\mathrm{m}=$ Meckel's cartilage, $\mathrm{pq}=$ palatoquadrate, $\mathrm{q}=$ quadrate. Scale bar $=200$ microns. Cartilage is stained blue (Alcian Blue), bone is stained red (Alizarin Red). 

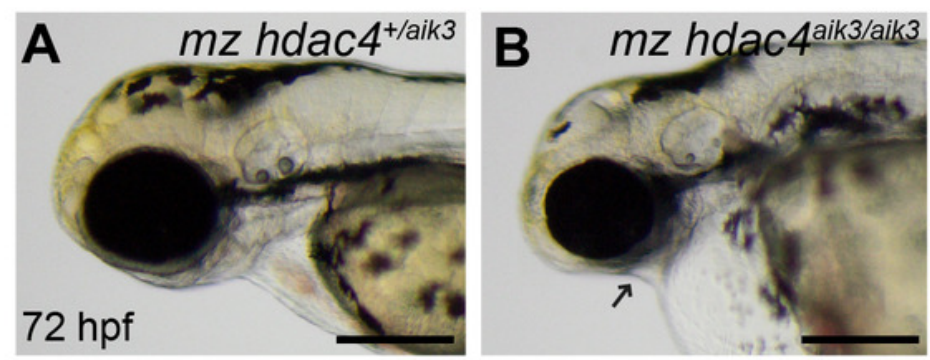

$m z$ hdac4 $^{+/ a i k 3}$

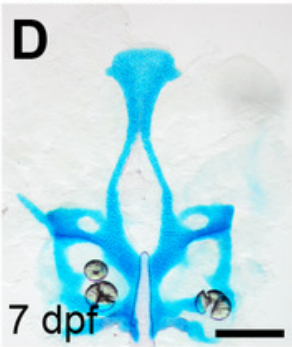

$m z$ hdac4 $^{+/ a i k 3}$

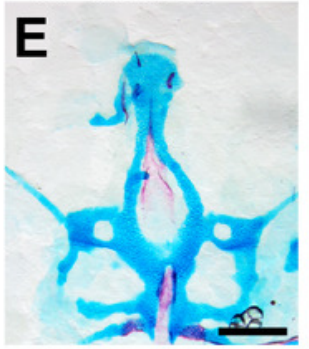

$m z$ hdac4 $4^{a i k 3 / a i k 3}$
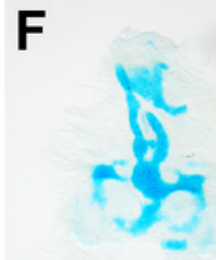

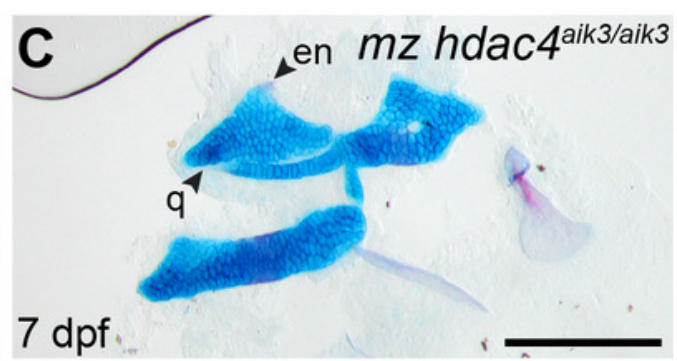

G

\begin{tabular}{|l|l|l|}
\hline & $\begin{array}{l}\text { m, pq } \\
\text { (arch 1) } \\
\text { defect }\end{array}$ & $\begin{array}{l}\text { neuro- } \\
\text { cranium } \\
\text { defect }\end{array}$ \\
\hline $\begin{array}{l}\mathbf{m Z} \\
\text { hdac4 }^{+/ a i k 3}\end{array}$ & $\begin{array}{l}\text { (10.2\%) } \\
\text { (10.2\%) }\end{array}$ & $\begin{array}{l}7 / 46 \\
(15.2 \%)\end{array}$ \\
\hline $\begin{array}{l}\mathbf{m Z} \\
\text { hdac4 }^{\text {aik3/aik3 }}\end{array}$ & $\begin{array}{l}15 / 58 \\
(25.9 \%)\end{array}$ & $\begin{array}{l}16 / 52 \\
(30.8 \%)\end{array}$ \\
\hline
\end{tabular}




\section{Figure 7}

Expression of hdac4 mRNA transcripts at different stages of embryonic and larval development.

A: RNA-seq mRNA levels of $h d a c 4$ transcripts from zygotic to larval day 5 stage. Bars indicate numbers of hdac4 transcripts per million transcripts at each stage. See http://www.ebi.ac.uk/gxa/experiments/E-ERAD-475 for Expression Atlas data. B: Expression of hdac4 mRNA at 4-8 cells, dorsal view. C: Expression of hdac4 mRNA at 16-32 cells, dorsal view. D: Expression of hdac4 mRNA at 256-512 cells, dorsal view. E: Expression of hdac4 mRNA at $75-90 \%$ epiboly, arrow indicates embryo dorsal to the yolk sac, lateral view. B-D: Scale bar $=200$ microns, E: Scale bar $=50$ microns. 


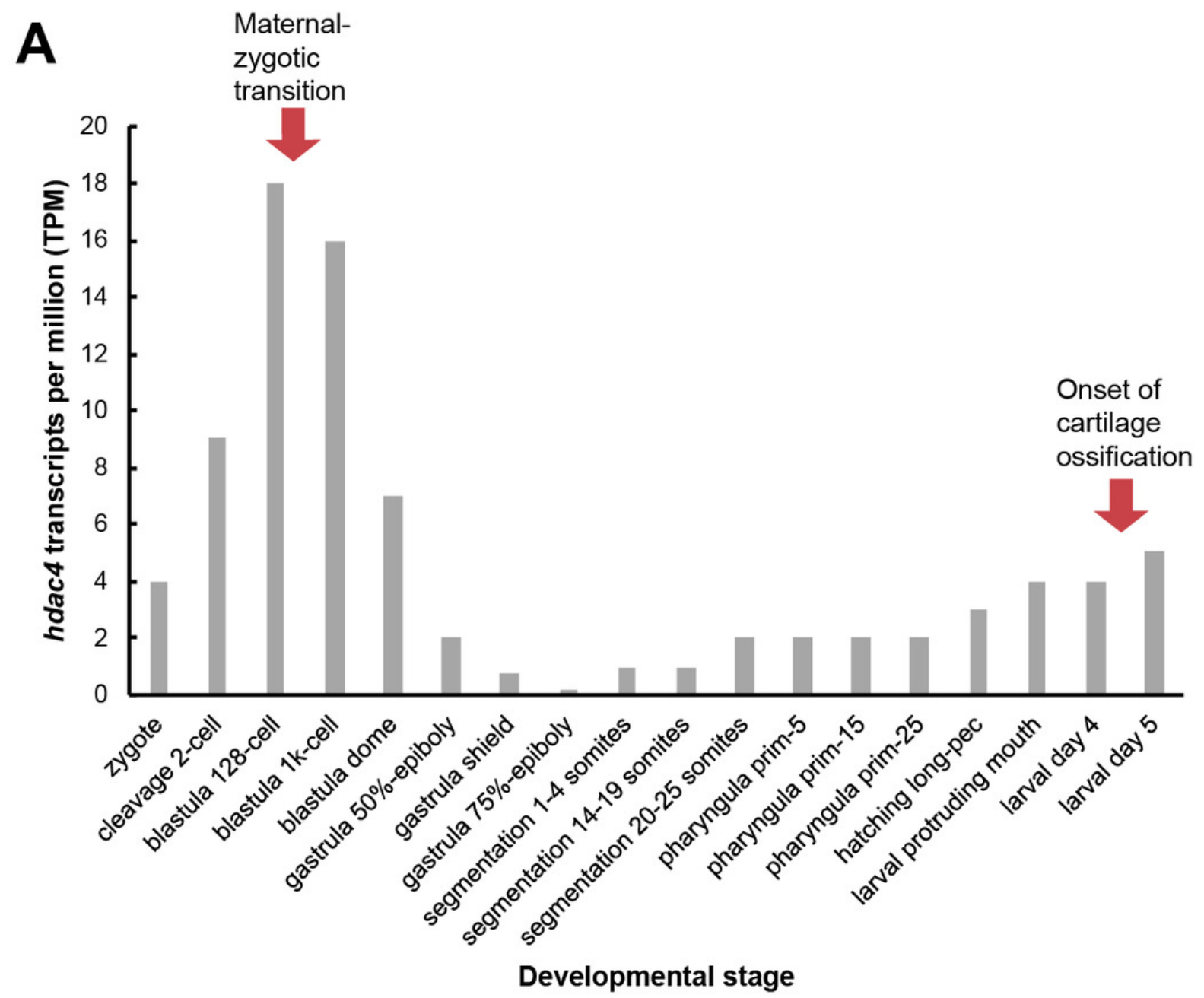

B

C
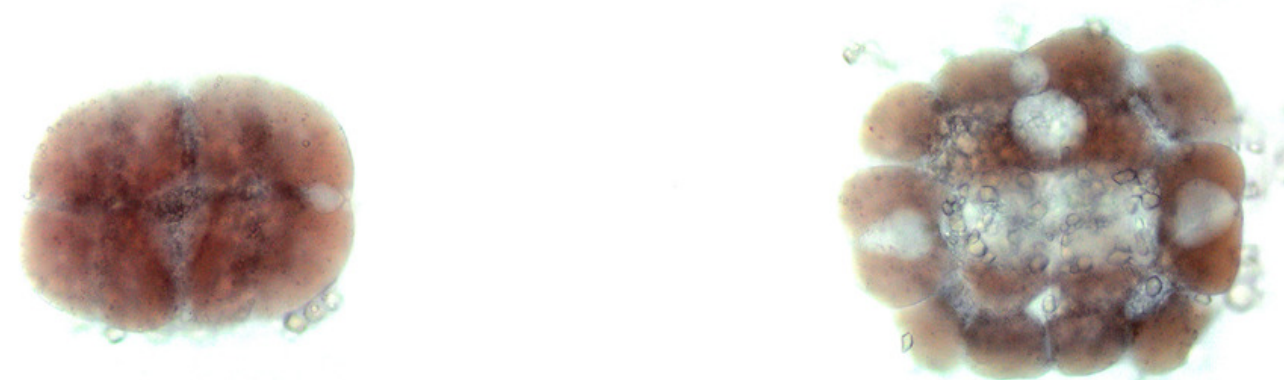

4-8 cells

16-32 cells

D
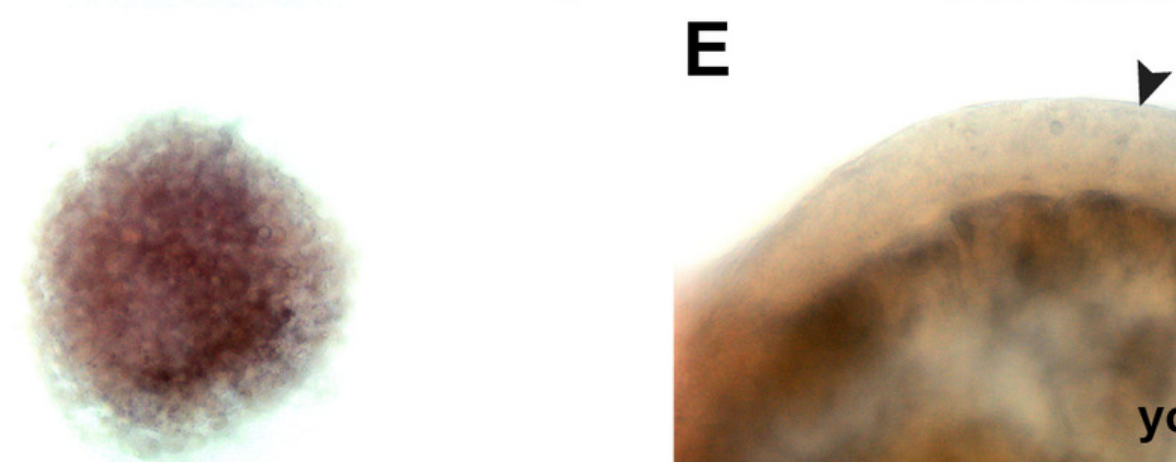

yolk 